\title{
1. Older women, public policy and work
}

When I first retired I found it really difficult because I'd worked full-time for 34 years. I found it difficult to not work and because I went from full-time work to not working at all, I believe these days you need to have a bit more transition. I suppose work is partly your reason for being, you know, like I don't have a family or anything, so work was the reason I got up in the morning and did things. [...] It, I suppose, validated me as a person and it was, I suppose, the way to earn money to live. (Jayne, Interview 5)

In this quote 'Jayne' (pseudonym) points out that professional women can share an orientation and commitment to work that characterizes an androcentric stereotype of productivity. However, by qualifying that she does not have a family, she implies that such a relationship to work is not usual for workers with caring and other responsibilities. While Jayne reports on her own experience, which may be a familiar experience to many professionals, hers is not necessarily representative of other older women's experiences of working. Women's experiences are not necessarily the same as or different to men's experiences, as there is not one story for women.

Reminiscent of Hakim's (1995) myths of women's employment, the themes of this chapter and ones that are developed in the following chapters are that it is both impossible and unhelpful to portray older women's experiences of working life as being uniform, to consider them as necessarily being strongly oriented to paid work, and inaccurate to characterize women's experiences as only being shaped by discrimination from employers and labour market disadvantage. Instead, this chapter and those that follow point to the need to resist reductionist efforts to develop policy for 'older workers' and an advocacy that emerges from productivist notions of the necessity of participation in the paid labour force and draw attention to areas that, perhaps as a consequence, have been overlooked or disregarded amid the present focus on the prolongation of working lives.

This chapter considers labour force participation of older women and maps trajectories and trends across countries. Specifically, it considers international trends in older women's labour force participation, how older women work and the kinds of work they do. The following chapter introduces key policy issues related to older women's labour force participation and changing understandings of retirement.

\section{OLDER WOMEN'S LABOUR FORCE PARTICIPATION - INTERNATIONAL COMPARISONS}

Levels of older women's labour force participation, in general, are markedly lower than is the case for men but, since 1960, women's labour force participation has been growing. As can be seen from Table 1.1, rates of labour force participation for women aged 55-64 for selected 
nations, between 1960 (where available) and 2015, show a long-term increase. However, this overall international trend belies the complexity of older women's relationships with the labour market. Notable, for instance, is that the trajectory has not always been upwards. Thus, for some countries in the 1980s, levels of women's participation fell back somewhat, perhaps as a consequence of the recession at the time. Also notable is the substantial variation in levels of participation between countries. For some nations, levels of older women's participation have grown dramatically, whereas for others these have grown barely at all. Substantial growth to high levels of participation in some nations contrasts with high growth but continuing modest participation elsewhere.

Considering variations in participation rates between women in this age group in these countries, Turkey, at 18 per cent in 2015, has the lowest and Iceland, at 83 per cent, has the highest. Comparing countries, starting well below the Organisation for Economic Co-operation and Development (OECD) average in 1970, Australia's participation rate had exceeded this by 2005. In 2015, Australia's participation rate, at 57 per cent, equalled that of the United Kingdom, and was only slightly less than that of Canada and the United States (both 59 per cent), but Australia saw a steeper increase after 1980. Notable is the vast difference between Australia and New Zealand, its near neighbour, which has witnessed a remarkable doubling of its labour force participation for women in this age group between 1990 (31 per cent) and 2015 (73 per cent).

Belgium, Germany, Hungary, the Netherlands, Spain and the Slovak Republic have also experienced substantial increases in older women's labour force participation. In other countries, such as Finland, Japan and Korea, participation was already relatively high in the 1980s and their consequent increases less steep.

Iceland's participation rate, at 83 per cent, was the highest for women in the 55-64 age group and saw slight fluctuations between 1995 and 2015 for which records were available. Sweden's labour force participation rate in 2015, although well below that for Iceland, rather exceeded those of some of its near neighbours: Denmark, Finland, Latvia and Lithuania. By contrast to the general picture of increasing older women's participation rates, Greece saw a shallow increase in participation rates whereas Turkey saw a decline during this period.

While many countries have seen sharp increases in levels of women's labour force participation in the 55-64 age group, for some levels remain comparatively low. Thus, in 2015 Austria, Greece, Hungary, Italy, Luxembourg, Mexico, Poland, Slovenia, Costa Rica, Brazil and South Africa each had rates in the 30-40 per cent range.

\section{HOW DO OLDER WOMEN WORK AND WHAT DO THEY DO?}

This section not only considers how older women work and what work they do, but also introduces the context for when they do this. In following chapters, we build on this to challenge ideas of the normative life course that shapes women's labour force participation.

Guillemard (2013) mapped transformations of the standardized biographical pattern of education, work and leisure that once organized the life course in industrialized society and noted that flexible working, flexible life courses and, as a result, new and less certain biographical pathways are multiplying. Kohli and Rein (1991) describe the transition to retirement as becoming 'fuzzier' (p. 22). 
Table 1.1 Labour force participation rates over time among women aged 55-64 in selected countries and overall OECD rates

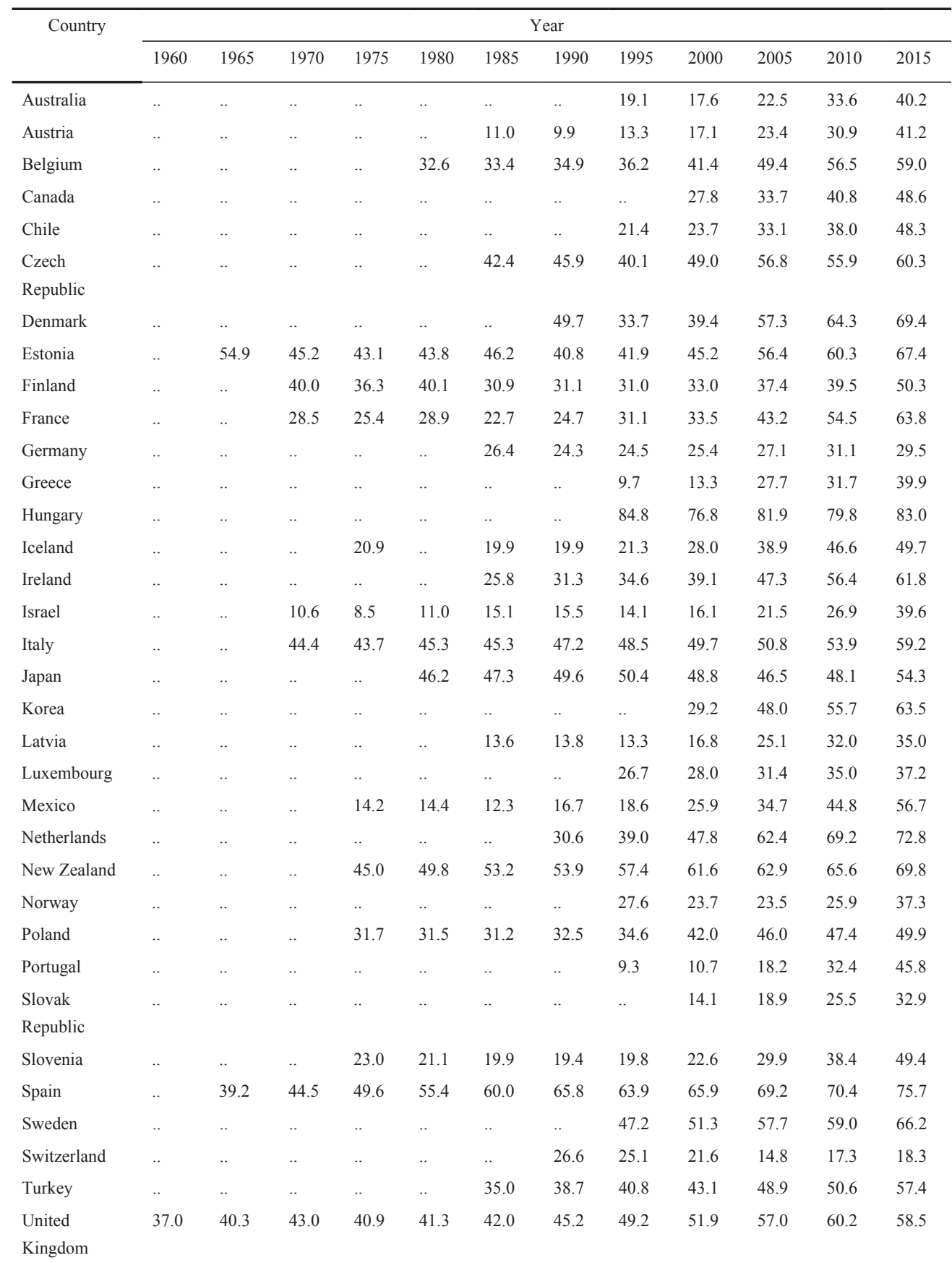




\begin{tabular}{|c|c|c|c|c|c|c|c|c|c|c|c|c|}
\hline \multirow[t]{2}{*}{ Country } & \multicolumn{12}{|c|}{ Year } \\
\hline & 1960 & 1965 & 1970 & 1975 & 1980 & 1985 & 1990 & 1995 & 2000 & 2005 & 2010 & 2015 \\
\hline United States & 37.0 & 30.9 & 37.9 & 35.0 & 37.0 & 34.1 & 36.0 & 36.2 & 38.6 & 43.5 & 47.8 & 52.2 \\
\hline $\begin{array}{l}\text { OECD } \\
\text { countries }\end{array}$ & 37.0 & 30.9 & 37.9 & 35.0 & 37.0 & 34.1 & 36.0 & 36.2 & 38.6 & 43.5 & 47.8 & 52.2 \\
\hline Colombia & .. & .. & .. & .. & .. &.. & .. &.. & .. & 35.8 & 43.0 & 49.7 \\
\hline Costa Rica & .. & .. &.. &.. &.. &.. & 13.3 & 15.9 & 21.0 & 30.1 & 32.9 & 38.9 \\
\hline Lithuania & .. & .. & .. & .. & .. & .. & .. &.. & 35.9 & 44.8 & 51.7 & 63.3 \\
\hline Brazil &.. & .. &.. &.. & .. &.. &.. &.. &.. & 41.5 &.. & 36.1 \\
\hline China &.. & .. &.. &.. &.. &.. & .. &.. & 47.2 &.. & 48.3 &.. \\
\hline India & .. & .. &.. &.. &.. &.. &.. &.. & 30.0 & 33.8 & 27.5 &.. \\
\hline Indonesia &.. & .. & .. &.. &.. & .. &.. &.. & 52.6 & 50.2 & 54.4 & 54.3 \\
\hline $\begin{array}{l}\text { Russian } \\
\text { Federation }\end{array}$ & .. & .. &.. & .. &.. &.. & .. & 23.1 & 27.8 & 38.8 & 39.9 & 41.2 \\
\hline South Africa &.. & .. & .. &.. &.. &.. &.. &.. &.. & 33.2 & 31.6 & 35.8 \\
\hline
\end{tabular}

Source: OECD.Stat.

They note the emergence of a range of in-between statuses connecting paid work and full retirement, however, as Earl and Taylor (2017) have pointed out, many retirements have long been blurred. For instance, portfolio retirement careers as experienced by women or those in non-career roles illustrate the diversity of patterns of retirement trajectories, although many of these were obscured until recently.

Giving impetus to these trends have been high-level efforts to remodel worker biographies so as to remove the demarcation between work and retirement. Thus, a discourse of active ageing 'is based on the simple notion that policy should take account of the continuity of life' (OECD, 1998, p. 84). Ostensibly, affording workers a greater degree of control over the nature and timing of their retirement aligns well with theoretical perspectives that draw a link between disengagement or continuity and worker wellbeing. Thus, continuity theory draws from the notion that people who are able to preserve a similar lifestyle in retirement will maintain their psychological wellbeing and, thus, some kind of bridge employment as a transition status before full retirement may serve to maintain a person's sense of structure and self-image through the enactment of activities that give life meaning. On the other hand, according to role theory, a status such as retirement, that does not align with social expectations, and particularly when it is entered involuntarily, may have adverse consequences for a person's wellbeing (Lytle et al., 2015).

A scaling back of working hours - commonly referred to as gradual retirement - has long been promoted as an approach that has appeal to older workers as they transition to retirement. Alongside notions of the general importance of participation in productive activities at older ages, providing older workers with the opportunity to downshift, by means of reduced working hours or levels of responsibility, is considered to be beneficial (Johnson et al., 2009). Proponents have pointed to supposed individual, organizational and societal benefits. According to Delsen and Reday-Mulvey (1996, p. 9) it 'constitutes a flexible extension of working life and should be promoted as such'. In Australia, the right to work flexibly featured prominently in a report from the Advisory Panel on the Economic Potential of Senior Australians (2011). 
Table 1.2 Labour force participation rates over time among women aged 65 and over in selected countries and overall OECD rates

\begin{tabular}{|c|c|c|c|c|c|c|c|c|c|c|c|c|}
\hline \multirow[t]{2}{*}{ Country } & \multicolumn{12}{|c|}{ Year } \\
\hline & 1960 & 1965 & 1970 & 1975 & 1980 & 1985 & 1990 & 1995 & 2000 & 2005 & 2010 & 2015 \\
\hline Australia &.. & .. & 3.7 & 3.9 & 2.8 & 2.1 & 2.4 & 2.6 & 3.1 & 4.2 & 6.9 & 8.6 \\
\hline Austria &.. &.. &.. &.. &.. &.. &.. & 2.4 & 1.7 & 1.6 & 3.5 & 3.6 \\
\hline Belgium &.. &.. &.. &.. &.. & 0.9 & 0.6 & 1.0 & 0.8 & 1.0 & 1.2 & 1.4 \\
\hline Canada & .. &.. &.. & .. & 3.8 & 3.9 & 3.5 & 3.3 & 3.3 & 5.0 & 7.4 & 9.5 \\
\hline Chile &.. &.. &.. &.. &.. &.. &.. &.. & 8.3 & 9.0 & 10.1 & 13.3 \\
\hline Czech &.. & .. &.. &.. &.. & .. &.. & 3.4 & 2.4 & 2.3 & 3.2 & 4.1 \\
\hline \multicolumn{13}{|l|}{ Republic } \\
\hline Denmark &.. & .. &.. &.. &.. & 3.2 & 3.4 & 0.9 & 1.4 & 2.7 & 3.0 & 3.6 \\
\hline Estonia &.. & .. &.. &.. &.. &.. & 19.1 & 6.1 & 11.8 & 15.4 & 14.8 & 22.5 \\
\hline Finland &.. & 3.8 & 11.0 & 8.5 & 5.6 & 4.8 & 3.4 & 2.0 & 1.6 & 3.2 & 5.0 & 7.9 \\
\hline France & .. & .. & 8.6 & 5.8 & 3.4 & 2.2 & 1.5 & 1.2 & 0.9 & 0.8 & 1.0 & 1.9 \\
\hline Germany &.. &.. & 6.1 & 4.6 & 3.2 & 2.3 & 2.2 & 1.6 & 1.5 & 2.2 & 2.8 & 4.1 \\
\hline Greece & .. & .. &.. & .. &.. & 5.4 & 4.5 & 3.7 & 2.8 & 1.9 & 2.1 & 2.1 \\
\hline Hungary & .. & .. &.. &.. &.. & .. &.. & 2.7 & 1.8 & 1.5 & 2.4 & 2.7 \\
\hline Iceland &.. &.. &.. &.. &.. & .. &.. & 31.8 & 22.6 & 22.2 & 27.8 & 29.6 \\
\hline Ireland &.. &.. &.. & 7.2 &.. & 3.9 & 3.4 & 3.0 & 3.1 & 3.3 & 4.5 & 5.4 \\
\hline Israel &.. & .. &.. &.. &.. & 6.3 & 6.9 & 6.4 & 4.3 & 6.0 & 7.2 & 13.0 \\
\hline Italy &.. & .. & 2.6 & 2.1 & 3.5 & 2.1 & 2.2 & 1.8 & 1.6 & 1.1 & 1.3 & 1.7 \\
\hline Japan &.. & .. & 17.9 & 15.3 & 15.5 & 15.5 & 16.2 & 15.6 & 14.4 & 12.7 & 13.3 & 15.3 \\
\hline Korea &.. &.. &.. &.. & 16.9 & 19.2 & 18.4 & 20.2 & 22.7 & 22.4 & 21.9 & 23.2 \\
\hline Latvia &.. &.. &.. &.. &.. &.. &.. &.. & 5.3 & 5.6 & 5.0 & 6.2 \\
\hline Luxembourg &.. & .. &.. &.. &.. & 2.0 & 1.1 & 1.1 & 1.2 & 0.4 & 1.9 & 2.2 \\
\hline Mexico &.. & .. &.. &.. &.. &.. &.. & 15.2 & 14.2 & 14.8 & 14.6 & 15.1 \\
\hline Netherlands & .. &.. &.. & 1.8 & 0.9 & 0.6 & 1.5 & 0.9 & 1.5 & 1.8 & 3.1 & 3.8 \\
\hline New Zealand &.. &.. &.. &.. &.. &.. & 3.6 & 2.9 & 4.4 & 8.0 & 12.4 & 16.9 \\
\hline Norway &.. &.. &.. & 12.1 & 12.7 & 13.6 & 12.0 & 9.0 & 8.5 & 11.5 & 13.7 & 14.8 \\
\hline Poland &.. &. &.. &.. &.. & .. &.. & 8.5 & 5.2 & 3.9 & 3.0 & 2.7 \\
\hline Portugal &.. &.. &.. & 10.7 & 8.6 & 7.2 & 7.8 & 9.4 & 13.0 & 13.2 & 12.5 & 7.2 \\
\hline Slovak &.. & .. &.. &.. &.. &.. &.. & 0.9 & 0.6 & 0.9 & 1.2 & 1.5 \\
\hline \multicolumn{13}{|l|}{ Republic } \\
\hline Slovenia &.. &.. &.. &.. &.. &.. &.. &.. & 6.5 & 5.5 & 5.2 & 3.1 \\
\hline Spain &.. &.. &.. & 6.3 & 4.0 & 2.3 & 1.7 & 1.4 & 1.0 & 1.1 & 1.5 & 1.4 \\
\hline Sweden &.. & 11.6 & 8.7 & 6.1 & 3.8 & 2.9 & 5.1 & 5.3 & 6.3 & 5.9 & 8.6 & 12.6 \\
\hline Switzerland &.. &.. &.. &.. &.. &.. &.. & 5.8 & 6.2 & 4.9 & 6.3 & 8.2 \\
\hline Turkey &.. &.. &.. &.. &.. &.. & 9.3 & 11.0 & 11.3 & 6.6 & 5.9 & 5.8 \\
\hline United &.. &.. &.. &.. &.. & 3.0 & 3.4 & 3.2 & 3.5 & 4.4 & 6.3 & 7.6 \\
\hline
\end{tabular}




\begin{tabular}{|c|c|c|c|c|c|c|c|c|c|c|c|c|}
\hline \multirow[t]{2}{*}{ Country } & \multicolumn{12}{|c|}{ Year } \\
\hline & 1960 & 1965 & 1970 & 1975 & 1980 & 1985 & 1990 & 1995 & 2000 & 2005 & 2010 & 2015 \\
\hline United States & 16.2 & 9.7 & 9.7 & 8.2 & 8.1 & 7.3 & 8.6 & 8.8 & 9.4 & 11.5 & 13.8 & 15.3 \\
\hline $\begin{array}{l}\text { OECD } \\
\text { countries }\end{array}$ & 16.2 & 9.7 & 9.7 & 8.2 & 8.2 & 6.9 & 6.9 & 6.1 & 5.8 & 7.4 & 8.6 & 9.9 \\
\hline Colombia & .. & .. & .. & .. & .. & .. & .. & .. & .. & 13.7 & 15.8 & 18.5 \\
\hline Costa Rica & .. & .. & .. & .. & .. & .. & 5.1 & 6.7 & 5.6 & 6.0 & 9.9 & 7.7 \\
\hline Lithuania & .. & .. & .. & .. & .. & .. & .. & .. & 6.2 & 2.6 & 3.2 & 4.8 \\
\hline Brazil & .. & .. & .. & .. & .. & .. & .. & .. & .. & 14.8 & .. & 8.2 \\
\hline China & .. & .. & .. & .. & .. & .. & .. & .. & 17.3 & .. & 15.1 & .. \\
\hline India & .. & .. & .. & .. & .. & .. & .. & .. & 11.0 & 12.9 & 11.3 & .. \\
\hline Indonesia & .. & .. & .. & .. & .. & .. & .. & .. & 29.4 & 28.2 & 28.9 & 27.2 \\
\hline $\begin{array}{l}\text { Russian } \\
\text { Federation }\end{array}$ &.. & .. & .. & .. &.. & .. & .. & 5.1 & 8.8 & 9.9 & 7.8 & 10.5 \\
\hline South Africa &.. & .. & .. & .. & .. & .. &.. & .. &.. & .. & 4.1 & 4.5 \\
\hline
\end{tabular}

Source: OECD.Stat.

Flexible work was defined as including part-time work, casual work, blocks of time to take leave and return to work, and working fewer hours. The report stated that 'Offering flexibility in the hours of work, types of work and the way the work is done, within the constraints of the particular workplace, is a key way to attract and retain older workers' (p. 9). Yet, while employment flexibility may suit employer needs, and according to Earl and Taylor (2015) this is open to doubt, its benefits for women are questionable. Such transitions may represent a narrowing of employment opportunities, resulting in employment with poorer terms and conditions and segmentation into lower-quality jobs (Smeaton et al., 2009). Notably, doubt has been cast on the universal desirability of a one-way street of flexibility in terms of reduced working hours. Bell and Rutherford (2013), for instance, note that many employees working reduced hours appear to consider their choices to be restricted and productive potential under-utilized. This raises the question of the influence of occupational mobility on older workers' wellbeing. Critics of phased or gradual models of retirement have pointed out that these assume a degree of control over the manner and timing of retirement likely to be absent for many workers (Taylor et al., 2016). Are more flexible forms of employment necessarily beneficial as has been claimed (Foster and Walker, 2015), or is there a need for a more nuanced consideration of older workers' transitions (Taylor and Earl, 2016)? For instance, research suggests that that downward occupational mobility in middle age has adverse consequences for women's health in later life (Lindsay et al., 2012). 
Table 1.3 Incidence of full-time employment over time for women aged 55-64 for selected countries

\begin{tabular}{|c|c|c|c|c|c|c|c|c|}
\hline \multirow[t]{2}{*}{ Country } & \multicolumn{8}{|c|}{ Year } \\
\hline & 1980 & 1985 & 1990 & 1995 & 2000 & 2005 & 2010 & 2015 \\
\hline Australia & .. &.. &.. &.. &.. & 57.6 & 60.4 & 61.3 \\
\hline Austria & .. &.. &.. & 68.4 & 74.0 & 61.0 & 60.6 & 60.2 \\
\hline Belgium &.. & 76.7 & 72.5 & 68.6 & 56.4 & 55.8 & 55.6 & 59.0 \\
\hline Canada & 72.1 & 70.2 & 68.8 & 67.1 & 69.9 & 70.7 & 71.6 & 74.6 \\
\hline Chile &.. &.. &.. &.. & 87.3 & 84.4 & 68.7 & 72.7 \\
\hline Colombia & .. &.. &.. &.. &.. & 64.8 & 65.1 & 65.8 \\
\hline Czech & .. &.. & .. & 67.6 & 75.8 & 89.2 & 89.7 & 90.7 \\
\hline \multicolumn{9}{|l|}{ Republic } \\
\hline Denmark & .. & 49.2 & 58.9 & 66.3 & 73.4 & 76.7 & 77.3 & 81.4 \\
\hline Estonia &.. &.. &.. &.. & 81.0 & 91.7 & 87.4 & 90.6 \\
\hline Finland & .. &.. & 81.3 & 81.9 & 78.2 & 80.6 & 79.0 & 83.9 \\
\hline France &.. & 73.5 & 70.2 & 67.0 & 66.1 & 72.1 & 73.0 & 73.2 \\
\hline Germany &.. & 68.9 & 61.3 & 59.1 & 55.6 & 54.3 & 58.0 & 58.9 \\
\hline Greece &.. & 89.2 & 89.2 & 85.5 & 90.9 & 88.5 & 86.6 & 85.4 \\
\hline Hungary &.. &.. &.. & 72.4 & 82.9 & 87.9 & 90.9 & 89.3 \\
\hline Iceland &.. &.. &.. & 61.3 & 64.7 & 69.5 & 73.4 & 76.7 \\
\hline Ireland &.. & 71.0 & 69.7 & 60.1 & 53.1 & 50.4 & 48.3 & 56.2 \\
\hline Israel &.. & .. &.. & 65.4 & 62.5 & 68.5 & 70.1 & 73.3 \\
\hline Italy &.. & 78.6 & 73.9 & 77.3 & 75.8 & 67.6 & 66.0 & 68.1 \\
\hline Japan & 69.7 & 68.3 & 67.5 & 67.6 & 64.9 & 63.0 & 59.5 & 57.5 \\
\hline Korea &.. &.. & 89.1 & 92.8 & 87.1 & 84.4 & 83.2 & 83.3 \\
\hline Latvia & .. &.. &.. & .. & 83.7 & 89.0 & 90.5 & 89.8 \\
\hline Lithuania &.. &.. &.. &.. & 80.2 & 85.8 & 88.5 & 87.9 \\
\hline Luxembourg &.. & 69.8 & 75.6 & 67.1 & 61.9 & 60.7 & 64.8 & 63.1 \\
\hline Mexico &.. &.. &.. & 55.3 & 65.2 & 63.9 & 64.2 & 64.6 \\
\hline Netherlands &.. & 36.5 & 29.2 & 27.8 & 31.0 & 30.9 & 32.2 & 34.3 \\
\hline New Zealand & .. &.. & 52.7 & 57.6 & 58.6 & 62.5 & 65.6 & 68.7 \\
\hline Norway &.. &.. & 48.0 & 52.4 & 58.4 & 63.2 & 64.9 & 70.5 \\
\hline Poland &.. &.. &.. &.. & 56.8 & 69.6 & 76.3 & 87.1 \\
\hline Portugal &.. &.. & 81.1 & 77.5 & 73.0 & 74.3 & 78.1 & 82.1 \\
\hline Slovak &.. &.. &.. & 87.0 & 85.4 & 82.9 & 91.9 & 90.6 \\
\hline \multicolumn{9}{|l|}{ Republic } \\
\hline Slovenia &.. &.. & .. &.. & 80.8 & 79.8 & 76.5 & 84.4 \\
\hline Spain &.. &.. & 84.8 & 83.1 & 82.5 & 76.5 & 79.0 & 79.1 \\
\hline Sweden &.. &.. &.. &.. & 74.2 & 78.8 & 80.9 & 85.0 \\
\hline Switzerland &.. &.. &.. &.. & 46.8 & 42.6 & 44.2 & 46.9 \\
\hline Turkey & .. &.. & 78.9 & 83.8 & 70.0 & 80.7 & 62.9 & 69.4 \\
\hline
\end{tabular}




\begin{tabular}{|c|c|c|c|c|c|c|c|c|}
\hline \multirow[t]{2}{*}{ Country } & \multicolumn{8}{|c|}{ Year } \\
\hline & 1980 & 1985 & 1990 & 1995 & 2000 & 2005 & 2010 & 2015 \\
\hline $\begin{array}{l}\text { United } \\
\text { Kingdom }\end{array}$ & .. & 46.1 & 45.1 & 44.3 & 47.6 & 51.8 & 53.4 & 57.1 \\
\hline $\begin{array}{l}\text { OECD } \\
\text { countries }\end{array}$ & 75.4 & 71.1 & 71.1 & 70.4 & 69.9 & 71.1 & 70.9 & 72.2 \\
\hline Costa Rica & .. & .. & .. & .. & .. & .. & 66.0 & 58.7 \\
\hline Brazil & .. & .. & .. & .. & .. & 53.7 & .. & 69.5 \\
\hline $\begin{array}{l}\text { Russian } \\
\text { Federation }\end{array}$ & .. & .. & .. & 91.1 & 77.0 & 87.3 & 91.0 & 90.5 \\
\hline South Africa & .. & .. & .. & .. & .. & .. & 82.5 & 81.8 \\
\hline
\end{tabular}

Source: OECD.Stat.

Table 1.3 shows rates of full-time employment for women in the 55-64 age range. Again, noticeable is the wide variability between countries. Thus, women in this age group in the Czech Republic, Estonia, the Russian Federation and the Slovak Republic have rates of full-time employment in excess of 90 per cent, compared with 34 per cent in the Netherlands. In fact, in a substantial number of countries, full-time employment is the norm for women in this age range. Clear trends in the incidence of full-time employment are difficult to discern across the range of countries. For the OECD countries as a whole, the rate of full-time employment has been steady at approximately 70 per cent since the 1980s. But, as an illustration, Denmark and Germany demonstrate divergent trends. While full-time employment is the predominant model, older women in some countries are much more likely to be in part-time roles than others. Thus, in Australia, Austria, Belgium, Germany, Ireland, Japan, Luxembourg, Mexico, the United Kingdom and Costa Rica, roughly two-fifths of women in this age group are classified as working part-time. 
Table $1.4 \quad$ Incidence of full-time employment over time for women aged in the 65 and over age range for selected countries

\begin{tabular}{|c|c|c|c|c|c|c|c|c|}
\hline \multirow[t]{2}{*}{ Country } & \multicolumn{8}{|c|}{ Year } \\
\hline & 1980 & 1985 & 1990 & 1995 & 2000 & 2005 & 2010 & 2015 \\
\hline Australia & .. &.. & .. &.. & .. & 38.4 & 39.0 & 38.4 \\
\hline Austria &.. &.. &.. & 39.6 & 66.3 & 37.4 & 26.4 & 23.5 \\
\hline Belgium &.. & 79.8 & 75.0 & 71.2 & 51.1 & 42.6 & 28.2 & 38.9 \\
\hline Canada & 45.2 & 43.4 & 37.1 & 39.0 & 39.5 & 41.5 & 42.3 & 46.7 \\
\hline Chile &.. &.. &.. & .. & 81.9 & 79.8 & 54.5 & 50.5 \\
\hline Colombia &.. & .. &.. &.. &.. & 57.2 & 48.4 & 48.1 \\
\hline Czech &.. & .. &.. &.. & .. & 41.3 & 41.9 & 43.3 \\
\hline \multicolumn{9}{|l|}{ Republic } \\
\hline Denmark &.. & 36.6 & 29.1 & 35.2 & 22.8 & 30.9 & 32.9 & 42.8 \\
\hline Estonia &.. &.. &.. &.. & 60.3 & 53.2 & 50.3 & 58.8 \\
\hline Finland &.. & .. & .. & .. & .. & 42.9 & 34.1 & 35.2 \\
\hline France &.. & 63.2 & 67.8 & 55.2 & 53.9 & 64.0 & 45.3 & 43.7 \\
\hline Germany &.. & 54.5 & 51.1 & 42.2 & 30.5 & 22.0 & 22.5 & 20.1 \\
\hline Greece &.. & 73.6 & 65.7 & 71.9 & 79.2 & 75.3 & 75.3 & 79.7 \\
\hline Hungary &.. & .. &.. &.. & 45.2 & 54.1 & 44.8 & 49.6 \\
\hline Ireland &.. & 70.0 & 67.0 & 69.2 & 56.6 & 34.6 & 26.7 & 33.6 \\
\hline Israel &.. &.. &.. & 28.7 & 27.2 & 29.0 & 32.4 & 39.8 \\
\hline Italy &.. & 68.4 & 69.5 & 76.0 & 76.5 & 65.7 & 62.9 & 59.1 \\
\hline Japan &.. & .. &.. & .. & .. & 53.0 & 48.6 & 44.4 \\
\hline Korea &.. & .. &.. & 85.3 & 77.2 & 72.1 & 62.0 & 57.8 \\
\hline Latvia &.. & .. &.. & .. & 66.0 & 67.9 & 75.8 & 69.9 \\
\hline Lithuania & .. & .. & .. &.. & 78.7 & 53.8 & 75.1 & 68.6 \\
\hline Luxembourg &.. & 80.1 & 48.9 & 75.0 & 58.8 & 58.2 & 33.8 & 28.4 \\
\hline Mexico &.. & .. &.. & 55.4 & 53.0 & 52.8 & 52.6 & 52.9 \\
\hline Netherlands &.. & 47.4 & 19.0 & 20.5 & 12.2 & 9.9 & 9.4 & 12.1 \\
\hline New Zealand &.. & .. & 39.1 & 35.5 & 35.9 & 38.5 & 41.4 & 46.4 \\
\hline Norway &.. &.. &.. &.. &.. & 45.3 & 37.9 & 42.7 \\
\hline Poland &.. &.. &.. &.. & .. & 38.1 & 43.1 & 51.2 \\
\hline Portugal &.. & .. & 71.6 & 68.3 & 46.2 & 45.2 & 40.9 & 44.5 \\
\hline Slovak &.. & .. &.. & 74.2 & 62.5 & 43.8 & 55.8 & 62.0 \\
\hline \multicolumn{9}{|l|}{ Republic } \\
\hline Slovenia & .. & .. &.. & .. & 72.1 & 48.1 & 54.3 & 52.4 \\
\hline Spain & .. & .. & 75.9 & 67.4 & 66.9 & 57.0 & 64.4 & 65.7 \\
\hline Sweden &.. & .. &.. & .. & .. &.. & 30.6 & 34.1 \\
\hline Switzerland &.. & .. &.. &.. & 22.6 & 19.2 & 16.5 & 14.6 \\
\hline Turkey &.. &.. & 70.0 & 81.0 & 70.0 & 81.3 & 53.6 & 65.4 \\
\hline
\end{tabular}




\begin{tabular}{|c|c|c|c|c|c|c|c|c|}
\hline \multirow[t]{2}{*}{ Country } & \multicolumn{8}{|c|}{ Year } \\
\hline & 1980 & 1985 & 1990 & 1995 & 2000 & 2005 & 2010 & 2015 \\
\hline $\begin{array}{l}\text { United } \\
\text { Kingdom }\end{array}$ & .. & 23.3 & 20.1 & 15.8 & 16.6 & 20.6 & 22.4 & 24.4 \\
\hline $\begin{array}{l}\text { OECD } \\
\text { countries }\end{array}$ & 44.2 & 46.7 & 48.5 & 53.7 & 54.2 & 53.0 & 50.8 & 51.5 \\
\hline Costa Rica & .. & .. & .. & .. & .. & .. & 58.8 & 37.4 \\
\hline Brazil & .. & .. & .. & .. & .. & 29.0 & .. & 54.9 \\
\hline $\begin{array}{l}\text { Russian } \\
\text { Federation }\end{array}$ & .. & .. & .. & 90.5 & 55.6 & 66.2 & 70.5 & 72.5 \\
\hline South Africa & .. & .. & .. & .. & .. & .. & .. & 68.8 \\
\hline
\end{tabular}

Source: OECD.Stat.

Table 1.4 shows the incidence of full-time employment for women in the 65 and over age group. By comparison with the 55-64 age group it is apparent that rates of full-time employment are generally lower for women aged 65 or older. Nonetheless, once again, there is marked variation between countries, with majorities of such women in a number of cases working full-time. Also notable is that there are divergent trends across countries, with some showing decreases and others increases in levels of full-time employment over time. Across the OECD countries, Table 1.4 shows that approximately half of women in this age group are working full-time.

Altogether, for both age groups of women, these figures are indicative of heterogeneity in terms of working hours within and between countries and that these patterns are not stable over time. This has consequences for the development of both organizational and public policy. 
Table 1.5 Working hours for women aged 55-64 over time in selected countries

\begin{tabular}{|c|c|c|c|c|c|c|c|c|c|}
\hline \multirow[t]{2}{*}{ Country } & \multirow{2}{*}{$\begin{array}{l}\text { Hour } \\
\text { bands }\end{array}$} & \multicolumn{8}{|c|}{ Year } \\
\hline & & 1980 & 1985 & 1990 & 1995 & 2000 & 2005 & 2010 & 2015 \\
\hline \multirow[t]{5}{*}{ Australia } & $1-19$ & .. & .. & .. &.. &.. & 23.2 & 21.2 & 19.0 \\
\hline & $20-29$ &.. & .. & .. &.. &.. & 19.2 & 18.4 & 19.8 \\
\hline & $30-34$ &.. &.. &.. &.. &.. & 9.6 & 11.5 & 13.1 \\
\hline & $35-39$ &.. &.. &.. &.. &.. & 19.8 & 21.4 & 20.7 \\
\hline & $40+$ &.. &.. &.. &.. &.. & 28.2 & 27.5 & 27.5 \\
\hline \multirow[t]{5}{*}{ Austria } & $1-19$ &.. &.. & .. & 18.6 & 11.6 & 21.4 & 20.9 & 20.6 \\
\hline & $20-29$ & .. &.. & .. & 12.9 & 14.4 & 17.6 & 18.5 & 19.2 \\
\hline & $30-34$ &.. &.. &.. & 6.7 & 9.1 & 7.8 & 9.3 & 10.2 \\
\hline & $35-39$ & .. &.. &.. & 11.2 & 12.4 & 11.0 & 12.1 & 11.9 \\
\hline & $40+$ & .. &.. & .. & 50.5 & 52.6 & 42.3 & 39.1 & 38.1 \\
\hline \multirow[t]{5}{*}{ Belgium } & $1-19$ &.. & 9.9 & 12.3 & 13.9 & 23.7 & 24.1 & 24.7 & 22.8 \\
\hline & $20-29$ & .. & 13.4 & 17.0 & 20.6 & 19.9 & 20.1 & 19.7 & 18.2 \\
\hline & $30-34$ & .. & 7.4 & 5.1 & 5.2 & 6.3 & 8.6 & 12.5 & 15.1 \\
\hline & $35-39$ & .. & 25.6 & 28.0 & 32.3 & 27.4 & 28.0 & 28.1 & 26.1 \\
\hline & $40+$ & .. & 43.8 & 37.5 & 28.0 & 22.7 & 19.2 & 14.9 & 17.8 \\
\hline \multirow[t]{5}{*}{ Canada } & $1-19$ & 14.8 & 16.4 & 17.0 & 18.1 & 14.2 & 13.2 & 13.2 & 11.4 \\
\hline & $20-29$ & 13.1 & 13.3 & 14.2 & 14.8 & 15.9 & 16.1 & 15.2 & 14.0 \\
\hline & $30-34$ & 8.0 & 8.8 & 8.9 & 9.0 & 10.1 & 9.7 & 10.7 & 10.5 \\
\hline & $35-39$ & 27.6 & 26.7 & 23.4 & 22.4 & 27.5 & 28.6 & 30.3 & 29.6 \\
\hline & $40+$ & 36.5 & 34.7 & 36.5 & 35.7 & 32.4 & 32.5 & 30.6 & 34.6 \\
\hline \multirow[t]{5}{*}{ Chile } & $1-19$ & .. &.. & .. & .. & 6.4 & 8.3 & 20.2 & 17.2 \\
\hline & 20-29 & .. &.. & .. &.. & 6.3 & 7.4 & 11.1 & 10.1 \\
\hline & $30-34$ & .. & .. & .. & .. & 8.0 & 7.7 & 5.7 & 5.1 \\
\hline & $35-39$ & .. & .. &.. &.. & 5.4 & 4.8 & 5.3 & 4.6 \\
\hline & $40+$ &.. &.. &.. &.. & 73.9 & 71.8 & 57.7 & 63.0 \\
\hline Czech & $1-19$ & .. &.. & .. & 14.3 & 10.4 & 3.5 & 3.8 & 2.7 \\
\hline \multirow[t]{4}{*}{ Republic } & $20-29$ & .. &.. &.. & 18.1 & 13.8 & 7.4 & 6.6 & 6.6 \\
\hline & $30-34$ &.. &.. &.. & 9.3 & 8.4 & 4.7 & 4.6 & 4.0 \\
\hline & $35-39$ &.. &.. &.. & 4.5 & 3.3 & 10.0 & 9.7 & 10.5 \\
\hline & $40+$ &.. &.. &.. & 53.8 & 64.2 & 74.5 & 75.4 & 76.1 \\
\hline \multirow[t]{5}{*}{ Denmark } & $1-19$ & .. & 18.9 & 17.6 & 14.4 & 10.2 & 7.9 & 8.0 & 7.3 \\
\hline & $20-29$ &.. & 31.9 & 23.2 & 18.9 & 16.4 & 15.4 & 14.8 & 11.3 \\
\hline & $30-34$ &.. & 10.9 & 12.0 & 14.0 & 18.8 & 18.8 & 21.7 & 20.3 \\
\hline & $35-39$ &.. & 6.4 & 37.5 & 44.2 & 41.1 & 43.1 & 47.5 & 51.2 \\
\hline & $40+$ &.. & 31.9 & 9.7 & 8.4 & 13.4 & 14.7 & 8.0 & 9.9 \\
\hline
\end{tabular}




\begin{tabular}{|c|c|c|c|c|c|c|c|c|c|}
\hline \multirow[t]{2}{*}{ Country } & \multirow{2}{*}{$\begin{array}{l}\text { Hour } \\
\text { bands }\end{array}$} & \multicolumn{8}{|c|}{ Year } \\
\hline & & 1980 & 1985 & 1990 & 1995 & 2000 & 2005 & 2010 & 2015 \\
\hline \multirow[t]{5}{*}{ Estonia } & $1-19$ & .. & .. & .. & .. & 6.0 & 1.9 & 4.6 & 4.4 \\
\hline & $20-29$ &.. & .. & .. & .. & 13.0 & 6.4 & 8.0 & 5.3 \\
\hline & $30-34$ &.. & .. & .. & .. & 5.4 & 2.8 & 4.6 & 3.3 \\
\hline & $35-39$ & .. & .. & .. & .. & 5.9 & 6.1 & 5.9 & 7.0 \\
\hline & $40+$ & .. & .. & .. & .. & 69.7 & 82.7 & 77.0 & 80.1 \\
\hline \multirow[t]{5}{*}{ Finland } & $1-19$ &.. & .. & 8.9 & 7.4 & 9.2 & 8.6 & 9.2 & 7.6 \\
\hline & $20-29$ & .. & .. & 9.8 & 10.6 & 12.6 & 10.8 & 11.8 & 8.5 \\
\hline & $30-34$ & .. & .. & 6.3 & 6.4 & 6.7 & 6.5 & 5.6 & 5.9 \\
\hline & $35-39$ &.. & .. & 54.5 & 53.2 & 45.4 & 49.5 & 51.3 & 58.2 \\
\hline & $40+$ &.. & .. & 20.5 & 22.3 & 26.1 & 24.7 & 22.0 & 19.8 \\
\hline \multirow[t]{5}{*}{ France } & $1-19$ &.. & 12.2 & 15.1 & 16.1 & 18.4 & 15.6 & 13.9 & 12.6 \\
\hline & $20-29$ &.. & 12.9 & 14.1 & 16.7 & 15.5 & 12.2 & 13.1 & 14.3 \\
\hline & $30-34$ &.. & 5.5 & 5.0 & 5.7 & 7.9 & 8.4 & 8.2 & 8.1 \\
\hline & $35-39$ &.. & 37.0 & 39.3 & 39.4 & 37.1 & 37.2 & 38.8 & 41.8 \\
\hline & $40+$ & .. & 32.4 & 26.5 & 22.1 & 21.1 & 26.5 & 26.0 & 23.2 \\
\hline \multirow[t]{5}{*}{ Germany } & $1-19$ &.. & 10.3 & 19.1 & 20.2 & 24.8 & 25.4 & 22.0 & 21.2 \\
\hline & $20-29$ &.. & 20.8 & 19.6 & 20.7 & 19.6 & 20.2 & 20.1 & 19.9 \\
\hline & $30-34$ &.. & 6.8 & 5.3 & 7.5 & 7.2 & 8.1 & 10.4 & 12.3 \\
\hline & $35-39$ &.. & 7.1 & 29.6 & 25.9 & 22.7 & 19.8 & 17.4 & 17.9 \\
\hline & $40+$ &.. & 55.0 & 26.3 & 25.7 & 25.8 & 26.5 & 30.1 & 28.7 \\
\hline \multirow[t]{5}{*}{ Greece } & $1-19$ & .. & 3.9 & 3.3 & 4.9 & 3.8 & 4.0 & 5.2 & 5.9 \\
\hline & $20-29$ &.. & 6.8 & 7.5 & 9.4 & 5.2 & 7.4 & 8.2 & 8.7 \\
\hline & $30-34$ &.. & 6.0 & 6.4 & 8.6 & 16.4 & 13.4 & 11.3 & 9.3 \\
\hline & $35-39$ & .. & 10.3 & 14.4 & 10.7 & 8.4 & 10.8 & 11.3 & 4.6 \\
\hline & $40+$ &.. & 73.0 & 68.4 & 66.4 & 66.2 & 64.4 & 64.0 & 71.5 \\
\hline \multirow[t]{5}{*}{ Hungary } & $1-19$ & .. & .. &.. & 9.0 & 4.7 & 2.2 & 1.9 & 1.6 \\
\hline & $20-29$ &.. & .. & .. & 18.6 & 12.3 & 9.8 & 7.3 & 9.1 \\
\hline & $30-34$ &.. &.. &.. & 8.5 & 6.8 & 3.8 & 5.1 & 5.0 \\
\hline & $35-39$ & .. & .. &.. & 2.1 & 2.4 & 2.1 & 2.7 & 1.2 \\
\hline & $40+$ & .. & .. & .. & 61.8 & 73.7 & 82.0 & 83.1 & 83.1 \\
\hline \multirow[t]{5}{*}{ Iceland } & $1-19$ & .. &.. &.. & 13.1 & 5.7 & 7.9 & 9.2 & 6.8 \\
\hline & $20-29$ & .. &.. & .. & 25.6 & 29.6 & 22.6 & 17.4 & 16.4 \\
\hline & $30-34$ & .. & .. & .. & 10.7 & 12.8 & 15.8 & 14.2 & 13.4 \\
\hline & $35-39$ &.. &.. &.. & 7.1 & 8.1 & 9.1 & 7.2 & 12.4 \\
\hline & $40+$ & .. & .. & .. & 43.5 & 43.9 & 44.6 & 52.0 & 50.9 \\
\hline
\end{tabular}




\begin{tabular}{|c|c|c|c|c|c|c|c|c|c|}
\hline \multirow[t]{2}{*}{ Country } & \multirow{2}{*}{$\begin{array}{l}\text { Hour } \\
\text { bands }\end{array}$} & \multicolumn{8}{|c|}{ Year } \\
\hline & & 1980 & 1985 & 1990 & 1995 & 2000 & 2005 & 2010 & 2015 \\
\hline \multirow[t]{5}{*}{ Ireland } & $1-19$ &.. & 12.0 & 12.1 & 16.1 & 20.5 & 22.4 & 23.5 & 18.1 \\
\hline & 20-29 &.. & 16.5 & 17.6 & 24.5 & 26.4 & 27.1 & 28.2 & 25.7 \\
\hline & $30-34$ &.. & 9.3 & 7.7 & 8.9 & 8.0 & 7.9 & 9.7 & 11.4 \\
\hline & $35-39$ &.. & 12.0 & 17.1 & 21.3 & 26.2 & 29.3 & 26.1 & 28.3 \\
\hline & $40+$ & .. & 50.1 & 45.4 & 29.2 & 18.9 & 13.2 & 12.5 & 16.5 \\
\hline \multirow[t]{5}{*}{ Israel } & $1-19$ &.. &.. &.. & 15.2 & 20.0 & 15.1 & 13.7 & 11.7 \\
\hline & 20-29 & .. & .. & .. & 19.4 & 17.5 & 16.5 & 16.3 & 15.0 \\
\hline & $30-34$ & .. & .. & .. & 10.7 & 9.0 & 10.7 & 10.9 & 8.0 \\
\hline & $35-39$ &.. &.. & .. & 11.4 & 10.1 & 9.6 & 9.9 & 9.5 \\
\hline & $40+$ &.. &.. &.. & 43.2 & 43.4 & 48.1 & 49.2 & 55.8 \\
\hline \multirow[t]{5}{*}{ Italy } & $1-19$ &.. & 7.3 & 10.4 & 10.1 & 10.5 & 13.1 & 13.1 & 11.6 \\
\hline & 20-29 &.. & 13.2 & 15.8 & 12.6 & 13.7 & 19.2 & 20.9 & 20.2 \\
\hline & $30-34$ &.. & 8.9 & 6.1 & 6.3 & 6.2 & 6.8 & 7.5 & 8.4 \\
\hline & $35-39$ & .. & 18.4 & 20.4 & 22.9 & 24.7 & 26.0 & 26.7 & 29.4 \\
\hline & $40+$ &.. & 52.2 & 47.4 & 48.1 & 45.0 & 34.8 & 31.8 & 30.4 \\
\hline \multirow[t]{5}{*}{ Japan } & $1-19$ & .. & .. & .. &.. &.. & 3.5 & 3.9 & 4.0 \\
\hline & 20-29 &.. &.. &.. &.. &.. & 8.7 & 9.6 & 10.1 \\
\hline & $30-34$ & 39.6 & 41.5 & 42.6 & 42.4 & 45.9 & 3.8 & 3.7 & 3.8 \\
\hline & $35-39$ &.. &.. & .. & .. & .. & 3.0 & 3.1 & 3.2 \\
\hline & $40+$ & 60.4 & 58.5 & 57.4 & 57.6 & 54.1 & 14.2 & 12.9 & 12.1 \\
\hline \multirow[t]{5}{*}{ Korea } & $1-19$ & .. &.. & 2.2 & 1.9 & 5.2 & 6.5 & 7.3 & 7.2 \\
\hline & $20-29$ & .. & .. & 8.8 & 5.3 & 7.7 & 9.1 & 9.6 & 9.5 \\
\hline & $30-34$ &.. & .. & 5.1 & 3.3 & 3.6 & 4.3 & 5.2 & 6.0 \\
\hline & $35-39$ & .. & .. & 12.4 & 9.2 & 8.7 & 9.2 & 9.1 & 8.7 \\
\hline & $40+$ & .. & .. & 71.6 & 80.2 & 74.7 & 70.9 & 68.9 & 68.6 \\
\hline \multirow[t]{5}{*}{ Latvia } & $1-19$ &.. &.. &.. &.. & 6.2 & 2.9 & 2.6 & 2.5 \\
\hline & $20-29$ & .. &.. &.. &.. & 10.1 & 8.1 & 6.9 & 7.6 \\
\hline & $30-34$ &.. &.. & .. &.. & 5.0 & 5.6 & 3.7 & 3.1 \\
\hline & $35-39$ & .. & .. &.. &.. & 7.4 & 4.4 & 2.7 & 2.4 \\
\hline & $40+$ &.. & .. & .. &.. & 71.3 & 79.0 & 84.1 & 84.3 \\
\hline \multirow[t]{5}{*}{ Lithuania } & $1-19$ &.. &.. &.. &.. & 4.4 & 2.9 & 2.5 & 3.5 \\
\hline & $20-29$ & .. &.. & .. &.. & 15.4 & 11.3 & 9.0 & 8.6 \\
\hline & $30-34$ & .. &.. &.. &.. & 7.0 & 5.5 & 4.5 & 3.3 \\
\hline & $35-39$ & .. & .. & .. &.. & 9.4 & 4.7 & 5.9 & 4.7 \\
\hline & $40+$ & .. &.. & .. &.. & 63.8 & 75.6 & 78.1 & 80.0 \\
\hline
\end{tabular}




\begin{tabular}{|c|c|c|c|c|c|c|c|c|c|}
\hline \multirow[t]{2}{*}{ Country } & \multirow{2}{*}{$\begin{array}{l}\text { Hour } \\
\text { bands }\end{array}$} & \multicolumn{8}{|c|}{ Year } \\
\hline & & 1980 & 1985 & 1990 & 1995 & 2000 & 2005 & 2010 & 2015 \\
\hline \multirow[t]{5}{*}{ Luxembourg } & $1-19$ &.. & 11.8 & 10.0 & 13.4 & 12.4 & 15.6 & 13.4 & 13.5 \\
\hline & $20-29$ &.. & 18.4 & 14.5 & 19.2 & 25.7 & 23.7 & 21.8 & 23.4 \\
\hline & $30-34$ &.. & 5.7 & 7.9 & 7.5 & 6.5 & 7.7 & 8.5 & 11.0 \\
\hline & $35-39$ &.. & 3.3 & 2.6 & 5.8 & 10.8 & 9.2 & 12.7 & 5.2 \\
\hline & $40+$ &.. & 60.8 & 65.1 & 54.1 & 44.5 & 43.8 & 43.6 & 46.9 \\
\hline \multirow[t]{5}{*}{ Mexico } & $1-19$ &.. &.. &.. & 30.6 & 19.4 & 21.5 & 21.5 & 22.3 \\
\hline & $20-29$ &.. &.. &.. & 14.1 & 15.4 & 14.6 & 14.2 & 13.1 \\
\hline & $30-34$ &.. & .. &.. & 5.6 & 6.3 & 7.6 & 6.5 & 6.7 \\
\hline & $35-39$ &.. & .. &.. & 9.1 & 9.9 & 9.9 & 8.3 & 8.4 \\
\hline & $40+$ &.. & .. &.. & 40.7 & 48.9 & 46.4 & 49.4 & 49.4 \\
\hline \multirow[t]{5}{*}{ Netherlands } & $1-19$ &.. & 40.3 & 50.6 & 46.8 & 43.7 & 42.4 & 36.5 & 31.1 \\
\hline & $20-29$ &.. & 23.3 & 20.1 & 25.1 & 25.2 & 26.7 & 31.3 & 34.6 \\
\hline & $30-34$ &.. & 6.7 & 9.2 & 10.0 & 10.5 & 13.9 & 14.0 & 15.6 \\
\hline & $35-39$ &.. & 4.1 & 8.9 & 5.9 & 10.9 & 9.8 & 8.9 & 9.3 \\
\hline & $40+$ &.. & 25.7 & 11.0 & 12.2 & 9.7 & 7.2 & 9.3 & 9.4 \\
\hline \multirow[t]{5}{*}{ New Zealand } & $1-19$ &.. &.. & 29.8 & 26.9 & 25.7 & 19.9 & 18.0 & 14.9 \\
\hline & $20-29$ &.. & .. & 17.4 & 15.5 & 15.7 & 17.6 & 16.3 & 16.4 \\
\hline & $30-34$ &.. & .. & 7.2 & 9.0 & 9.6 & 9.9 & 11.5 & 11.2 \\
\hline & $35-39$ &.. & .. & 10.0 & 9.0 & 10.8 & 9.7 & 10.9 & 9.9 \\
\hline & $40+$ &.. & .. & 35.6 & 39.5 & 38.3 & 43.0 & 43.3 & 47.5 \\
\hline \multirow[t]{5}{*}{ Norway } & $1-19$ &.. &.. & 31.5 & 29.0 & 24.2 & 20.7 & 19.6 & 18.0 \\
\hline & $20-29$ &.. & .. & 20.5 & 18.5 & 17.4 & 16.1 & 15.4 & 11.6 \\
\hline & $30-34$ & .. & .. & 7.9 & 8.9 & 8.7 & 10.1 & 11.9 & 12.9 \\
\hline & $35-39$ &.. &.. & 33.1 & 37.1 & 42.3 & 48.1 & 46.6 & 49.5 \\
\hline & $40+$ & .. & .. & 7.1 & 6.5 & 7.4 & 5.0 & 6.4 & 8.0 \\
\hline \multirow[t]{5}{*}{ Poland } & $1-19$ &.. & .. &.. &.. & 21.7 & 11.3 & 9.9 & 3.8 \\
\hline & $20-29$ &.. & .. &.. & .. & 21.5 & 19.2 & 13.8 & 9.1 \\
\hline & $30-34$ &.. & .. &.. & .. & 10.1 & 7.0 & 7.2 & 4.2 \\
\hline & $35-39$ &.. & .. &.. & .. & 4.3 & 3.6 & 4.2 & 4.1 \\
\hline & $40+$ &.. & .. &.. & .. & 42.4 & 58.9 & 65.0 & 78.7 \\
\hline \multirow[t]{5}{*}{ Portugal } & $1-19$ & .. &.. & 9.1 & 11.3 & 11.0 & 11.6 & 10.6 & 10.8 \\
\hline & $20-29$ &.. &.. & 7.9 & 11.2 & 16.0 & 14.1 & 11.3 & 7.1 \\
\hline & $30-34$ & .. &.. & 6.1 & 5.3 & 10.6 & 7.8 & 6.5 & 3.2 \\
\hline & $35-39$ &.. &.. & 10.3 & 12.5 & 15.0 & 20.8 & 21.7 & 15.1 \\
\hline & $40+$ &.. &.. & 66.6 & 59.7 & 47.4 & 45.7 & 49.9 & 63.8 \\
\hline
\end{tabular}




\begin{tabular}{|c|c|c|c|c|c|c|c|c|c|}
\hline \multirow[t]{2}{*}{ Country } & \multirow{2}{*}{$\begin{array}{l}\text { Hour } \\
\text { bands }\end{array}$} & \multicolumn{8}{|c|}{ Year } \\
\hline & & 1980 & 1985 & 1990 & 1995 & 2000 & 2005 & 2010 & 2015 \\
\hline Slovak & $1-19$ &.. &.. &.. & 3.9 & 3.6 & 6.0 & 2.1 & 3.0 \\
\hline \multirow[t]{4}{*}{ Republic } & 20-29 &.. &.. &.. & 9.2 & 11.1 & 11.1 & 6.0 & 6.4 \\
\hline & $30-34$ &.. & .. &.. & 5.3 & 4.7 & 3.8 & 2.3 & 1.6 \\
\hline & $35-39$ &.. &.. &.. & 1.9 & 1.2 & 16.5 & 22.5 & 24.3 \\
\hline & $40+$ &.. &.. &.. & 79.7 & 79.4 & 62.6 & 67.1 & 64.7 \\
\hline \multirow[t]{5}{*}{ Slovenia } & $1-19$ &.. &.. &.. & .. & 9.9 & 10.7 & 10.3 & 5.4 \\
\hline & $20-29$ &.. &.. &.. &.. & 9.2 & 9.6 & 13.2 & 10.2 \\
\hline & $30-34$ &.. & .. &.. & .. & 2.0 & 4.1 & 2.6 & 1.4 \\
\hline & $35-39$ &.. &.. &.. & .. & 3.8 & 1.2 & 2.0 & 0.1 \\
\hline & $40+$ &.. &.. &.. & .. & 75.0 & 74.5 & 71.9 & 82.9 \\
\hline \multirow[t]{5}{*}{ Spain } & $1-19$ &.. &.. & 7.0 & 8.4 & 8.4 & 11.7 & 9.5 & 9.8 \\
\hline & $20-29$ &.. &.. & 8.1 & 8.5 & 9.1 & 11.7 & 11.5 & 11.1 \\
\hline & $30-34$ &.. & .. & 7.8 & 6.3 & 6.5 & 7.7 & 6.4 & 5.3 \\
\hline & $35-39$ &.. &.. & 10.9 & 12.9 & 16.3 & 24.2 & 27.5 & 30.7 \\
\hline & $40+$ &.. & .. & 66.2 & 63.9 & 59.7 & 44.6 & 45.2 & 43.1 \\
\hline \multirow[t]{5}{*}{ Sweden } & $1-19$ &.. &.. &.. & .. & 6.1 & 4.6 & 4.4 & 3.8 \\
\hline & $20-29$ &.. &.. &.. & .. & 19.7 & 16.6 & 14.7 & 11.3 \\
\hline & $30-34$ &.. & .. &.. & .. & 15.3 & 18.3 & 19.5 & 17.7 \\
\hline & $35-39$ &.. & .. & .. & .. & 15.0 & 15.5 & 16.5 & 17.7 \\
\hline & $40+$ & .. & .. & .. & .. & 43.9 & 44.9 & 45.0 & 49.5 \\
\hline \multirow[t]{5}{*}{ Switzerland } & $1-19$ &.. &.. &.. & .. & 31.9 & 33.3 & 30.5 & 25.9 \\
\hline & $20-29$ &.. &.. &.. & .. & 21.3 & 24.0 & 25.3 & 27.2 \\
\hline & $30-34$ &.. & .. &.. &.. & 8.3 & 9.5 & 10.9 & 13.4 \\
\hline & $35-39$ &.. & .. &.. & .. & 5.4 & 6.1 & 6.8 & 7.4 \\
\hline & $40+$ &.. & .. & .. & .. & 33.1 & 27.0 & 26.6 & 26.1 \\
\hline \multirow[t]{5}{*}{ Turkey } & $1-19$ &.. &.. & 7.0 & 4.1 & 11.0 & 6.2 & 14.4 & 13.2 \\
\hline & $20-29$ &.. &.. & 14.1 & 12.1 & 19.0 & 13.1 & 22.7 & 17.4 \\
\hline & $30-34$ &.. &.. & 9.1 & 5.1 & 7.6 & 6.8 & 3.2 & 8.0 \\
\hline & $35-39$ &.. & .. & 6.0 & 7.8 & 11.7 & 12.2 & 10.3 & 12.5 \\
\hline & $40+$ &.. &.. & 63.8 & 70.9 & 50.8 & 61.7 & 49.4 & 48.9 \\
\hline United & $1-19$ & .. & 32.7 & 31.8 & 32.9 & 31.0 & 26.0 & 25.1 & 21.6 \\
\hline \multirow[t]{4}{*}{ Kingdom } & $20-29$ &.. & 21.2 & 23.1 & 22.7 & 21.5 & 22.3 & 21.5 & 21.3 \\
\hline & $30-34$ & .. & 8.0 & 8.1 & 7.6 & 8.1 & 9.7 & 10.7 & 11.6 \\
\hline & $35-39$ &.. & 21.8 & 22.6 & 20.4 & 21.1 & 21.8 & 22.4 & 22.3 \\
\hline & $40+$ & .. & 16.3 & 14.4 & 16.4 & 18.3 & 20.3 & 20.3 & 23.2 \\
\hline
\end{tabular}




\begin{tabular}{|c|c|c|c|c|c|c|c|c|c|}
\hline \multirow[t]{2}{*}{ Country } & \multirow{2}{*}{$\begin{array}{l}\text { Hour } \\
\text { bands }\end{array}$} & \multicolumn{8}{|c|}{ Year } \\
\hline & & 1980 & 1985 & 1990 & 1995 & 2000 & 2005 & 2010 & 2015 \\
\hline \multirow[t]{5}{*}{ United States } & $1-19$ &.. &.. &.. &.. &.. & .. &.. & .. \\
\hline & $20-29$ &.. &.. &.. & .. & .. & .. &.. &.. \\
\hline & $30-34$ &.. &.. &.. & .. &.. &.. &.. &.. \\
\hline & $35-39$ &.. &.. &.. & .. & .. & .. & .. &.. \\
\hline & $40+$ &.. &.. &.. &.. & .. & .. & .. & .. \\
\hline \multirow{5}{*}{$\begin{array}{l}\text { OECD } \\
\text { countries }\end{array}$} & $1-19$ & 5.4 & 8.8 & 9.0 & 9.9 & 10.3 & 12.8 & 12.7 & 12.0 \\
\hline & $20-29$ & 6.0 & 9.7 & 9.8 & 9.9 & 10.3 & 16.1 & 16.4 & 15.8 \\
\hline & $30-34$ & 20.7 & 18.4 & 17.9 & 17.6 & 17.8 & 8.5 & 8.7 & 8.7 \\
\hline & $35-39$ & 8.9 & 11.3 & 12.4 & 11.5 & 11.2 & 15.2 & 15.2 & 15.7 \\
\hline & $40+$ & 59.0 & 51.8 & 50.9 & 51.0 & 50.3 & 47.4 & 46.9 & 47.9 \\
\hline \multirow[t]{5}{*}{ Costa Rica } & $1-19$ &.. &.. &.. & .. &.. & .. & 20.3 & 30.4 \\
\hline & $20-29$ &.. &.. &.. & .. & .. & .. & 13.7 & 10.9 \\
\hline & $30-34$ &.. &.. &.. & .. & .. & .. & 1.7 & 4.8 \\
\hline & $35-39$ &.. &.. &.. &.. & .. & .. & 8.0 & 4.1 \\
\hline & $40+$ &.. &.. &.. & .. & .. & .. & 56.2 & 49.8 \\
\hline European & $1-19$ &.. & 17.4 & 18.8 & 19.6 & 19.3 & 17.6 & 16.1 & 14.5 \\
\hline \multirow[t]{4}{*}{ Union 28} & $20-29$ &.. & 18.0 & 16.9 & 17.9 & 18.0 & 17.3 & 17.0 & 16.4 \\
\hline & $30-34$ &.. & 7.3 & 6.7 & 7.2 & 8.8 & 9.0 & 9.4 & 9.5 \\
\hline & $35-39$ &.. & 19.0 & 25.1 & 23.2 & 20.1 & 21.5 & 21.6 & 22.5 \\
\hline & $40+$ &.. & 38.3 & 32.6 & 32.0 & 33.9 & 34.7 & 35.9 & 37.1 \\
\hline \multirow[t]{5}{*}{ Brazil } & $1-19$ &.. &.. & .. & .. & .. & 25.9 &.. & 15.0 \\
\hline & $20-29$ &.. &.. &.. & .. &.. & 20.4 &.. & 15.5 \\
\hline & $30-34$ &.. &.. &.. &.. &.. & 11.0 &.. & 10.2 \\
\hline & $35-39$ & .. &.. & .. & .. & .. & 4.7 &.. & 4.9 \\
\hline & $40+$ &.. &.. &.. &.. &.. & 37.9 &.. & 54.4 \\
\hline Russian & $1-19$ &.. &.. &.. & 5.4 & 14.1 & 7.9 & 5.1 & 5.7 \\
\hline \multirow[t]{4}{*}{ Federation } & $20-29$ &.. &.. &.. & 3.4 & 8.9 & 4.8 & 3.9 & 3.8 \\
\hline & $30-34$ &.. &.. &.. &.. & .. & .. &.. &.. \\
\hline & $35-39$ &.. &.. &.. & 89.5 & 69.6 & 86.9 & 90.6 & 90.2 \\
\hline & $40+$ &.. &.. &.. & 1.6 & 7.4 & 0.4 & 0.4 & 0.3 \\
\hline \multirow[t]{5}{*}{ South Africa } & $1-19$ &.. &.. &.. &.. & .. & .. & 8.3 & 10.5 \\
\hline & $20-29$ &.. &.. &.. & .. &.. &.. & 9.2 & 7.7 \\
\hline & $30-34$ &.. &.. &.. & .. & .. &.. & 5.1 & 4.7 \\
\hline & $35-39$ &.. &.. &.. & .. &.. &.. & 9.6 & 7.9 \\
\hline & $40+$ & .. & .. &.. & .. &.. &.. & 67.9 & 69.2 \\
\hline
\end{tabular}




\begin{tabular}{|c|c|c|c|c|c|c|c|c|c|}
\hline \multirow[t]{2}{*}{ Country } & \multirow{2}{*}{$\begin{array}{l}\text { Hour } \\
\text { bands }\end{array}$} & \multicolumn{8}{|c|}{ Year } \\
\hline & & 1980 & 1985 & 1990 & 1995 & 2000 & 2005 & 2010 & 2015 \\
\hline \multirow[t]{5}{*}{ Colombia } & $1-19$ &.. & .. & .. & .. & .. & 21.1 & 20.0 & 17.9 \\
\hline & $20-29$ &.. & .. & .. & .. & .. & 14.1 & 14.9 & 16.3 \\
\hline & $30-34$ & .. & .. & .. & .. & .. & 9.4 & 8.1 & 8.9 \\
\hline & $35-39$ & .. & .. & .. & .. & .. & 4.9 & 5.2 & 5.1 \\
\hline & $40+$ & .. & .. & .. & .. & .. & 50.5 & 51.8 & 51.8 \\
\hline
\end{tabular}

Source: OECD.Stat.

Table 1.5 considers working hours among women aged 55-64 in more detail. Here, working hours are broken down into bandings. Immediately noticeable in this table is the relatively high proportion of women who work 40 hours or more per week in a number of these countries. Also noticeable are wide variations between the countries. Thus, in the case of Latvia over three-quarters of women in the age group work 40 or more hours per week whereas this is the case for fewer than a tenth of equivalent women in Norway. By contrast, for most countries, small minorities of women in this age group work between 1 and 19 hours per week. 
Table 1.6 Working hours for women aged 65 and over, over time in selected countries

\begin{tabular}{|c|c|c|c|c|c|c|c|c|c|}
\hline \multirow[t]{2}{*}{ Country } & \multirow[t]{2}{*}{ Hour bands } & \multicolumn{8}{|c|}{ Year } \\
\hline & & 1980 & 1985 & 1990 & 1995 & 2000 & 2005 & 2010 & 2015 \\
\hline \multirow[t]{5}{*}{ Australia } & $1-19$ &.. &.. &.. & .. &.. & 44.5 & 42.5 & 42.5 \\
\hline & $20-29$ &.. &.. & .. & .. &.. & 17.2 & 18.5 & 19.1 \\
\hline & $30-34$ &.. &.. & .. & .. &.. & 8.2 & 9.2 & 8.9 \\
\hline & $35-39$ &.. & .. & .. & .. &.. & 11.5 & 10.9 & 11.1 \\
\hline & $40+$ &.. &.. &.. & .. & .. & 18.8 & 19.0 & 18.4 \\
\hline \multirow[t]{5}{*}{ Austria } & $1-19$ &.. &.. &.. & 44.6 & 17.9 & 49.4 & 56.5 & 62.7 \\
\hline & $20-29$ & .. & .. & .. & 15.9 & 15.8 & 13.2 & 17.1 & 13.8 \\
\hline & $30-34$ &.. &.. & .. & 9.0 & 10.0 & 4.9 & 5.9 & 5.6 \\
\hline & $35-39$ &.. &.. &.. & 3.5 & 10.8 & 3.4 & 5.2 & 4.5 \\
\hline & $40+$ &.. &.. & .. & 27.0 & 45.5 & 29.1 & 15.4 & 13.4 \\
\hline \multirow[t]{5}{*}{ Belgium } & $1-19$ &.. & 8.1 & 15.4 & 19.8 & 35.9 & 29.3 & 59.1 & 41.9 \\
\hline & $20-29$ & .. & 10.7 & 11.4 & 17.3 & 13.0 & 28.1 & 12.8 & 19.2 \\
\hline & $30-34$ &.. & 6.6 & 8.0 & 5.4 & 3.5 &.. & 0.9 & 6.9 \\
\hline & $35-39$ &.. & 9.9 & 0.7 & 18.7 & 5.9 & 3.5 & 3.2 & 8.5 \\
\hline & $40+$ & .. & 64.7 & 64.5 & 38.7 & 41.6 & 39.1 & 24.1 & 23.5 \\
\hline \multirow[t]{5}{*}{ Canada } & $1-19$ & 33.4 & 36.0 & 43.5 & 40.9 & 42.6 & 35.6 & 36.0 & 34.0 \\
\hline & 20-29 & 21.4 & 20.5 & 19.4 & 20.1 & 17.8 & 22.9 & 21.7 & 19.3 \\
\hline & $30-34$ & 11.1 & 9.3 & 8.3 & 9.9 & 10.8 & 9.2 & 9.2 & 9.7 \\
\hline & $35-39$ & 11.5 & 10.9 & 8.8 & 6.9 & 8.7 & 10.0 & 11.4 & 13.3 \\
\hline & $40+$ & 22.6 & 23.3 & 20.1 & 22.2 & 20.1 & 22.3 & 21.7 & 23.7 \\
\hline \multirow[t]{5}{*}{ Chile } & $1-19$ &.. &.. & .. & .. & 9.0 & 10.9 & 28.4 & 34.2 \\
\hline & $20-29$ &.. &.. & .. & .. & 9.1 & 9.3 & 17.1 & 15.3 \\
\hline & $30-34$ &.. &.. & .. & .. & 8.5 & 7.6 & 5.0 & 5.5 \\
\hline & $35-39$ &.. &.. &.. & .. & 5.5 & 3.6 & 5.2 & 4.7 \\
\hline & $40+$ &.. &.. & .. & .. & 67.8 & 68.5 & 44.4 & 40.3 \\
\hline \multirow[t]{5}{*}{ Czech Republic } & $1-19$ &.. &.. &.. & .. &.. & 26.0 & 26.7 & 29.8 \\
\hline & $20-29$ & .. & .. & .. & .. &.. & 32.6 & 31.4 & 26.9 \\
\hline & $30-34$ &.. &.. &.. & .. &.. & 8.3 & 10.4 & 8.1 \\
\hline & $35-39$ &.. &.. & .. & .. &.. & 4.5 & 4.5 & 2.6 \\
\hline & $40+$ & .. & .. & .. & .. &.. & 28.5 & 27.0 & 32.6 \\
\hline \multirow[t]{5}{*}{ Denmark } & $1-19$ &.. & 31.4 & 53.2 & 39.4 & 56.4 & 52.8 & 50.2 & 42.7 \\
\hline & $20-29$ & .. & 31.9 & 17.8 & 25.4 & 20.8 & 16.3 & 17.0 & 14.5 \\
\hline & $30-34$ &.. & 11.6 & 7.0 & 19.4 & 6.4 & 7.3 & 6.1 & 11.4 \\
\hline & $35-39$ &.. & 2.5 & 12.0 & 15.8 & 8.3 & 15.9 & 20.3 & 26.6 \\
\hline & $40+$ &.. & 22.6 & 9.9 & .. & 8.1 & 7.7 & 6.5 & 4.8 \\
\hline
\end{tabular}




\begin{tabular}{|c|c|c|c|c|c|c|c|c|c|}
\hline \multirow[t]{2}{*}{ Country } & \multirow[t]{2}{*}{ Hour bands } & \multicolumn{8}{|c|}{ Year } \\
\hline & & 1980 & 1985 & 1990 & 1995 & 2000 & 2005 & 2010 & 2015 \\
\hline \multirow[t]{5}{*}{ Estonia } & $1-19$ & .. &.. &.. & .. & 14.4 & 18.2 & 17.3 & 17.4 \\
\hline & $20-29$ & .. &.. &.. & .. & 25.3 & 28.7 & 32.4 & 24.2 \\
\hline & $30-34$ & .. &.. & .. & .. & 9.0 & 3.8 & 5.6 & 9.2 \\
\hline & $35-39$ &.. &.. &.. & .. & 5.6 & 7.3 & 9.2 & 8.2 \\
\hline & $40+$ & .. &.. &.. & .. & 45.7 & 42.1 & 35.6 & 41.0 \\
\hline \multirow[t]{5}{*}{ Finland } & $1-19$ & .. &.. &.. & .. &.. & 42.9 & 48.0 & 48.2 \\
\hline & 20-29 &.. &.. &.. &.. & .. & 14.3 & 17.9 & 16.6 \\
\hline & $30-34$ & .. &.. & .. & .. &.. & 14.3 & 6.5 & 4.0 \\
\hline & $35-39$ & .. &.. &.. & .. & .. & .. & 12.2 & 14.6 \\
\hline & $40+$ & .. & .. &.. & .. & .. & 28.6 & 15.4 & 16.6 \\
\hline \multirow[t]{5}{*}{ France } & $1-19$ & .. & 20.5 & 16.6 & 24.4 & 29.8 & 22.1 & 37.6 & 39.0 \\
\hline & 20-29 & .. & 14.7 & 11.6 & 14.5 & 16.3 & 14.0 & 17.1 & 17.2 \\
\hline & $30-34$ & .. & 6.9 & 4.4 & 6.8 & 7.2 & 10.9 & 4.5 & 5.2 \\
\hline & $35-39$ & .. & 13.0 & 20.9 & 13.2 & 13.3 & 17.6 & 15.7 & 15.4 \\
\hline & $40+$ & .. & 44.9 & 46.4 & 41.2 & 33.4 & 35.5 & 25.1 & 23.2 \\
\hline \multirow[t]{5}{*}{ Germany } & $1-19$ & .. & 23.4 & 32.7 & 41.6 & 57.3 & 65.4 & 65.2 & 69.2 \\
\hline & $20-29$ & .. & 22.0 & 16.3 & 16.2 & 12.2 & 12.6 & 12.3 & 10.8 \\
\hline & $30-34$ & .. & 9.6 & 5.8 & 6.6 & 5.1 & 3.7 & 4.0 & 4.5 \\
\hline & $35-39$ & .. & 2.6 & 6.5 & 8.5 & 4.3 & 2.6 & 3.1 & 2.8 \\
\hline & $40+$ & .. & 42.3 & 38.8 & 27.1 & 21.0 & 15.7 & 15.5 & 12.7 \\
\hline \multirow[t]{5}{*}{ Greece } & $1-19$ & .. & 14.8 & 20.3 & 12.1 & 9.5 & 7.1 & 16.2 & 11.5 \\
\hline & 20-29 & .. & 11.0 & 14.1 & 16.0 & 11.2 & 17.6 & 8.4 & 8.8 \\
\hline & $30-34$ & .. & 7.0 & 7.4 & 8.2 & 19.4 & 13.3 & 12.0 & 14.8 \\
\hline & $35-39$ & .. & 15.6 & 13.5 & 11.5 & 11.8 & 3.3 & 10.3 & 2.6 \\
\hline & $40+$ & .. & 51.6 & 44.8 & 52.2 & 48.0 & 58.7 & 53.1 & 62.3 \\
\hline \multirow[t]{5}{*}{ Hungary } & $1-19$ & .. &.. &.. & .. & 22.6 & 14.2 & 18.9 & 20.5 \\
\hline & $20-29$ & .. &.. & .. & .. & 32.2 & 31.7 & 36.3 & 30.0 \\
\hline & $30-34$ & .. &.. &.. & .. & 5.2 & 6.5 & 11.0 & 6.1 \\
\hline & $35-39$ & .. & .. & .. & .. & 1.4 & 1.4 & 1.9 & 1.2 \\
\hline & $40+$ & .. & .. & .. & .. & 38.5 & 46.2 & 32.0 & 42.2 \\
\hline \multirow[t]{5}{*}{ Iceland } & $1-19$ & .. &.. & .. & .. & .. & .. &.. & .. \\
\hline & $20-29$ &.. &.. &.. &.. &.. &.. &.. & .. \\
\hline & $30-34$ &.. &.. & .. & .. & .. & .. & .. & .. \\
\hline & $35-39$ & .. &.. & .. & .. & .. & .. & .. & .. \\
\hline & $40+$ & .. &.. &.. & .. & .. & .. & .. & .. \\
\hline
\end{tabular}




\begin{tabular}{|c|c|c|c|c|c|c|c|c|c|}
\hline \multirow[t]{2}{*}{ Country } & \multirow[t]{2}{*}{ Hour bands } & \multicolumn{8}{|c|}{ Year } \\
\hline & & 1980 & 1985 & 1990 & 1995 & 2000 & 2005 & 2010 & 2015 \\
\hline \multirow[t]{5}{*}{ Ireland } & $1-19$ & .. & 19.2 & 19.4 & 15.6 & 30.3 & 40.0 & 47.2 & 47.4 \\
\hline & $20-29$ & .. & 11.3 & 13.5 & 15.7 & 13.1 & 25.4 & 26.1 & 19.0 \\
\hline & $30-34$ & .. & 10.4 & 9.1 & 8.9 & 10.1 & 6.9 & 5.1 & 6.7 \\
\hline & $35-39$ & .. & 3.4 & 7.6 & 10.3 & 15.3 & 10.4 & 9.2 & 10.5 \\
\hline & $40+$ & .. & 55.6 & 50.3 & 49.5 & 31.3 & 17.4 & 12.4 & 16.4 \\
\hline \multirow[t]{5}{*}{ Israel } & $1-19$ &.. & .. & .. & 32.0 & 38.9 & 43.7 & 44.9 & 35.6 \\
\hline & $20-29$ & .. &.. &.. & 39.4 & 33.9 & 27.3 & 22.8 & 24.6 \\
\hline & $30-34$ & .. & .. & .. & 9.5 & 10.4 & 5.9 & 9.1 & 7.4 \\
\hline & $35-39$ & .. &.. &.. & 6.9 & 4.5 & 6.2 & 6.0 & 7.8 \\
\hline & $40+$ & .. &.. &.. & 12.2 & 12.2 & 16.8 & 17.3 & 24.6 \\
\hline \multirow[t]{5}{*}{ Italy } & $1-19$ &.. & 15.8 & 14.5 & 9.7 & 10.3 & 17.7 & 21.2 & 19.1 \\
\hline & 20-29 & .. & 13.9 & 15.2 & 14.2 & 13.2 & 16.6 & 15.9 & 21.8 \\
\hline & $30-34$ & .. & 10.3 & 7.5 & 7.4 & 7.8 & 7.2 & 8.6 & 8.1 \\
\hline & $35-39$ & .. & 12.0 & 12.0 & 15.5 & 13.4 & 13.6 & 7.4 & 8.7 \\
\hline & $40+$ & .. & 47.9 & 50.7 & 53.1 & 55.2 & 44.9 & 46.9 & 42.3 \\
\hline \multirow[t]{5}{*}{ Japan } & $1-19$ &.. & .. &.. & .. &.. & 6.4 & 7.3 & 7.7 \\
\hline & 20-29 & .. &.. & .. & .. &.. & 9.2 & 9.8 & 10.7 \\
\hline & $30-34$ & .. &.. &.. &.. &.. & 3.3 & 3.3 & 3.3 \\
\hline & $35-39$ & .. &.. &.. & .. & .. & 2.8 & 2.5 & 2.4 \\
\hline & $40+$ & .. &.. &.. & .. & .. & 11.6 & 10.4 & 9.0 \\
\hline \multirow[t]{5}{*}{ Korea } & $1-19$ &.. &.. & .. & 3.9 & 9.3 & 13.5 & 21.0 & 27.0 \\
\hline & $20-29$ & .. & .. & .. & 10.8 & 13.5 & 14.5 & 17.0 & 15.2 \\
\hline & $30-34$ & .. &.. & .. & 5.8 & 6.3 & 5.5 & 6.2 & 7.6 \\
\hline & $35-39$ & .. &.. &.. & 14.9 & 12.4 & 14.6 & 12.5 & 11.5 \\
\hline & $40+$ & .. &.. &.. & 64.7 & 58.5 & 52.0 & 43.3 & 38.7 \\
\hline \multirow[t]{5}{*}{ Latvia } & $1-19$ &.. &.. &.. & .. & 13.7 & 22.1 & 4.3 & 9.7 \\
\hline & $20-29$ & .. &.. &.. &.. & 20.3 & 10.0 & 20.0 & 20.4 \\
\hline & $30-34$ & .. &.. &.. &.. & 4.6 & 3.6 & 3.8 & 4.7 \\
\hline & $35-39$ & .. &.. & .. & .. & 11.2 & 5.8 & 4.0 & 2.4 \\
\hline & $40+$ & .. &.. &.. &.. & 50.2 & 58.4 & 68.0 & 62.8 \\
\hline \multirow[t]{5}{*}{ Lithuania } & $1-19$ &.. & .. &.. & .. & 7.5 & 16.8 & 8.7 & 13.2 \\
\hline & $20-29$ & .. & .. & .. & .. & 13.8 & 29.4 & 16.2 & 18.3 \\
\hline & $30-34$ & .. & .. &.. & .. & 8.6 & 4.7 & 4.1 & 7.2 \\
\hline & $35-39$ & .. & .. & .. & .. & 8.8 & 2.2 & 7.6 & 2.3 \\
\hline & $40+$ & .. & .. &.. & .. & 61.3 & 46.9 & 63.4 & 59.0 \\
\hline
\end{tabular}




\begin{tabular}{|c|c|c|c|c|c|c|c|c|c|}
\hline \multirow[t]{2}{*}{ Country } & \multirow[t]{2}{*}{ Hour bands } & \multicolumn{8}{|c|}{ Year } \\
\hline & & 1980 & 1985 & 1990 & 1995 & 2000 & 2005 & 2010 & 2015 \\
\hline \multirow[t]{5}{*}{ Luxembourg } & $1-19$ & .. & 8.5 & 17.7 & .. & 25.9 & .. & 37.2 & 39.4 \\
\hline & $20-29$ & .. & 11.4 & 33.4 & 23.1 & 15.3 & 41.8 & 28.9 & 32.2 \\
\hline & $30-34$ & .. & 2.8 & 3.8 & 7.7 & 19.7 & .. &.. & .. \\
\hline & $35-39$ & .. & 2.8 & .. & 7.7 & .. & 16.4 & .. & .. \\
\hline & $40+$ & .. & 74.4 & 45.1 & 61.5 & 39.1 & 41.8 & 33.8 & 28.4 \\
\hline \multirow[t]{5}{*}{ Mexico } & $1-19$ &.. &.. &.. & 31.6 & 28.6 & 30.1 & 29.5 & 30.3 \\
\hline & $20-29$ & .. &.. &.. & 13.0 & 18.5 & 17.1 & 17.9 & 16.8 \\
\hline & $30-34$ & .. &.. &.. & 5.8 & 4.8 & 6.1 & 6.4 & 6.1 \\
\hline & $35-39$ &.. &.. &.. & 10.6 & 10.9 & 10.5 & 8.7 & 8.5 \\
\hline & $40+$ & .. &.. & .. & 39.0 & 37.2 & 36.1 & 37.5 & 38.3 \\
\hline \multirow[t]{5}{*}{ Netherlands } & $1-19$ &.. & 36.2 & 60.9 & 61.6 & 84.0 & 74.8 & 76.7 & 71.8 \\
\hline & 20-29 & .. & 16.3 & 20.2 & 17.5 & 3.8 & 15.3 & 13.9 & 16.1 \\
\hline & $30-34$ & .. & 12.1 & 2.7 & 4.6 & 2.6 & 2.1 & 5.5 & 4.4 \\
\hline & $35-39$ & .. & 4.7 & 8.6 & .. & 1.5 & 1.2 & 0.9 & 2.7 \\
\hline & $40+$ & .. & 30.7 & 7.6 & 16.3 & 8.1 & 6.5 & 3.0 & 5.0 \\
\hline \multirow[t]{5}{*}{ New Zealand } & $1-19$ & .. &.. & 44.9 & 50.0 & 47.6 & 43.0 & 39.0 & 35.4 \\
\hline & 20-29 & .. &.. & 15.9 & 14.5 & 16.5 & 18.5 & 19.6 & 18.3 \\
\hline & $30-34$ & .. &.. & 5.8 & 4.8 & 6.8 & 6.5 & 10.1 & 11.2 \\
\hline & $35-39$ & .. & .. & 2.9 & 6.5 & 3.9 & 4.0 & 6.4 & 6.5 \\
\hline & $40+$ & .. &.. & 30.4 & 24.2 & 25.2 & 28.0 & 24.8 & 28.7 \\
\hline \multirow[t]{5}{*}{ Norway } & $1-19$ & .. & .. &.. & .. & .. & 38.6 & 45.4 & 41.1 \\
\hline & $20-29$ & .. & .. & .. & .. & .. & 16.1 & 16.7 & 16.2 \\
\hline & $30-34$ & .. &.. &.. &.. &.. & 6.4 & 6.3 & 7.8 \\
\hline & $35-39$ & .. &.. & .. & .. & .. & 30.8 & 26.9 & 30.1 \\
\hline & $40+$ & .. &.. &.. & .. & .. & 8.1 & 4.6 & 4.8 \\
\hline \multirow[t]{5}{*}{ Poland } & $1-19$ & .. & .. &.. &.. & .. & 35.4 & 28.5 & 22.0 \\
\hline & $20-29$ &.. &.. &.. &.. &.. & 26.4 & 28.3 & 26.8 \\
\hline & $30-34$ & .. &.. &.. &.. & .. & 10.9 & 14.9 & 10.7 \\
\hline & $35-39$ &.. & .. & .. & .. & .. & 4.3 & 4.8 & 4.6 \\
\hline & $40+$ & .. &.. & .. &.. & .. & 23.0 & 23.5 & 35.9 \\
\hline \multirow[t]{5}{*}{ Portugal } & $1-19$ & .. & .. & 13.6 & 17.2 & 25.9 & 33.6 & 35.2 & 42.0 \\
\hline & $20-29$ &.. &.. & 12.1 & 14.5 & 27.9 & 21.2 & 23.9 & 13.4 \\
\hline & $30-34$ & .. &.. & 10.5 & 7.3 & 12.2 & 13.8 & 13.2 & 6.5 \\
\hline & $35-39$ & .. &.. & 6.8 & 6.2 & 6.6 & 4.6 & 3.2 & 6.9 \\
\hline & $40+$ & .. &.. & 57.1 & 54.7 & 27.4 & 26.8 & 24.5 & 31.1 \\
\hline
\end{tabular}




\begin{tabular}{|c|c|c|c|c|c|c|c|c|c|}
\hline \multirow[t]{2}{*}{ Country } & \multirow[t]{2}{*}{ Hour bands } & \multicolumn{8}{|c|}{ Year } \\
\hline & & 1980 & 1985 & 1990 & 1995 & 2000 & 2005 & 2010 & 2015 \\
\hline \multirow[t]{5}{*}{ Slovak Republic } & $1-19$ & .. & .. & .. & 3.2 & .. & 31.3 & 18.6 & 16.9 \\
\hline & $20-29$ & .. & .. &.. & 22.6 & 37.5 & 25.0 & 25.6 & 21.1 \\
\hline & $30-34$ & .. & .. & .. & 3.2 & 6.3 & 9.4 & 7.0 & 8.5 \\
\hline & $35-39$ & .. & .. & .. & .. & .. & 3.1 & 14.0 & 5.6 \\
\hline & $40+$ & .. & .. &.. & 71.0 & 56.3 & 31.3 & 34.9 & 47.9 \\
\hline \multirow[t]{5}{*}{ Slovenia } & $1-19$ & .. & .. & .. & .. & 9.9 & 27.1 & 24.5 & 23.0 \\
\hline & $20-29$ & .. & .. & .. & .. & 17.9 & 24.8 & 21.2 & 24.6 \\
\hline & $30-34$ & .. & .. & .. & .. & 8.6 & 7.8 & 10.8 & 6.9 \\
\hline & $35-39$ & .. & .. & .. & .. & 4.5 & 3.0 & 7.0 & 3.5 \\
\hline & $40+$ & .. & .. & .. & .. & 59.0 & 37.2 & 36.5 & 42.0 \\
\hline \multirow[t]{5}{*}{ Spain } & $1-19$ & .. & .. & 12.0 & 21.9 & 18.0 & 26.9 & 20.7 & 19.9 \\
\hline & $20-29$ & .. & .. & 12.1 & 10.7 & 15.0 & 16.0 & 14.9 & 14.4 \\
\hline & $30-34$ & .. & .. & 10.1 & 6.4 & 5.5 & 8.8 & 5.7 & 6.8 \\
\hline & $35-39$ & .. & .. & 9.5 & 9.6 & 9.9 & 10.5 & 12.7 & 14.8 \\
\hline & $40+$ & .. & .. & 56.4 & 51.5 & 51.5 & 37.7 & 46.0 & 44.2 \\
\hline \multirow[t]{5}{*}{ Sweden } & $1-19$ & .. & .. &.. & .. & .. & .. & 50.5 & 44.1 \\
\hline & $20-29$ &.. & .. & .. & .. & .. & .. & 18.8 & 21.8 \\
\hline & $30-34$ & .. & .. & .. & .. & .. & .. & 9.0 & 8.0 \\
\hline & $35-39$ & .. & .. & .. & .. & .. &.. & 5.5 & 5.6 \\
\hline & $40+$ & .. & .. & .. & .. & .. & .. & 16.2 & 20.6 \\
\hline \multirow[t]{5}{*}{ Switzerland } & $1-19$ &.. & .. &.. & .. & 62.4 & 67.5 & 68.3 & 74.6 \\
\hline & $20-29$ & .. & .. & .. & .. & 15.0 & 13.3 & 15.2 & 10.8 \\
\hline & $30-34$ & .. & .. & .. & .. & 6.3 & 2.3 & 2.6 & 2.5 \\
\hline & $35-39$ & .. & .. & .. & .. & 1.4 & 1.7 & 2.2 & 2.4 \\
\hline & $40+$ & .. & .. & .. & .. & 14.9 & 15.3 & 11.7 & 9.7 \\
\hline \multirow[t]{5}{*}{ Turkey } & $1-19$ & .. & .. & 10.4 & 6.0 & 16.6 & 6.6 & 18.7 & 16.6 \\
\hline & $20-29$ & .. & .. & 19.6 & 13.0 & 13.5 & 12.0 & 27.7 & 18.0 \\
\hline & $30-34$ & .. & .. & 15.2 & 8.4 & 13.0 & 8.4 & 1.8 & 7.3 \\
\hline & $35-39$ & .. & .. & 8.3 & 7.9 & 8.5 & 13.3 & 12.7 & 17.1 \\
\hline & $40+$ & .. & .. & 46.5 & 64.8 & 48.4 & 59.6 & 39.2 & 41.0 \\
\hline \multirow[t]{5}{*}{ United Kingdom } & $1-19$ & .. & 59.8 & 60.8 & 67.4 & 65.4 & 58.6 & 57.6 & 55.5 \\
\hline & $20-29$ & .. & 16.9 & 19.1 & 16.4 & 18.1 & 20.8 & 20.0 & 20.1 \\
\hline & $30-34$ & .. & 6.8 & 5.3 & 5.3 & 8.0 & 5.6 & 6.2 & 7.1 \\
\hline & $35-39$ & .. & 6.5 & 5.3 & 4.0 & 4.5 & 7.6 & 6.1 & 7.7 \\
\hline & $40+$ & .. & 10.1 & 9.4 & 6.9 & 4.0 & 7.4 & 10.1 & 9.5 \\
\hline
\end{tabular}




\begin{tabular}{|c|c|c|c|c|c|c|c|c|c|}
\hline \multirow[t]{2}{*}{ Country } & \multirow[t]{2}{*}{ Hour bands } & \multicolumn{8}{|c|}{ Year } \\
\hline & & 1980 & 1985 & 1990 & 1995 & 2000 & 2005 & 2010 & 2015 \\
\hline \multirow[t]{5}{*}{ United States } & $1-19$ & .. & .. & .. & .. & .. & .. & .. & .. \\
\hline & $20-29$ & .. &.. &.. & .. & .. & .. & .. & .. \\
\hline & $30-34$ & .. & .. & .. & .. & .. & .. & .. & .. \\
\hline & $35-39$ & .. &.. &.. & .. & .. & .. & .. & .. \\
\hline & $40+$ & .. &.. &.. & .. & .. & .. & .. & .. \\
\hline \multirow[t]{5}{*}{ OECD countries } & $1-19$ & 32.5 & 31.2 & 29.8 & 26.8 & 26.2 & 25.4 & 27.5 & 27.5 \\
\hline & $20-29$ & 23.3 & 22.1 & 21.8 & 19.4 & 19.5 & 21.6 & 21.8 & 21.0 \\
\hline & $30-34$ & 6.5 & 8.3 & 8.2 & 7.5 & 8.4 & 8.5 & 7.9 & 8.2 \\
\hline & $35-39$ & 9.7 & 9.2 & 9.2 & 8.8 & 9.1 & 8.8 & 8.6 & 8.1 \\
\hline & $40+$ & 28.1 & 29.2 & 31.0 & 37.4 & 36.7 & 35.8 & 34.3 & 35.2 \\
\hline \multirow[t]{5}{*}{ Costa Rica } & $1-19$ & .. & .. & .. & .. & .. & .. & 35.4 & 42.3 \\
\hline & $20-29$ & .. &.. &.. & .. & .. & .. & 5.8 & 20.3 \\
\hline & $30-34$ & .. & .. & .. & .. & .. & .. & 6.8 & 3.9 \\
\hline & $35-39$ & .. & .. & .. & .. & .. & .. & 9.0 & 2.8 \\
\hline & $40+$ & .. & .. & .. & .. & .. & .. & 43.0 & 30.7 \\
\hline \multirow[t]{5}{*}{ European Union 28} & $1-19$ & .. & 32.0 & 32.9 & 35.8 & 30.3 & 38.3 & 43.0 & 46.0 \\
\hline & $20-29$ & .. & 17.3 & 15.8 & 15.4 & 22.1 & 20.7 & 20.0 & 18.1 \\
\hline & $30-34$ & .. & 8.4 & 6.8 & 6.6 & 11.5 & 9.4 & 8.0 & 7.2 \\
\hline & $35-39$ & .. & 7.8 & 8.8 & 8.4 & 8.7 & 7.5 & 6.3 & 7.0 \\
\hline & $40+$ & .. & 34.5 & 35.8 & 33.8 & 27.4 & 24.1 & 22.8 & 21.6 \\
\hline \multirow[t]{5}{*}{ Brazil } & $1-19$ & .. & .. &.. & .. & .. & 52.2 & .. & 26.5 \\
\hline & $20-29$ & .. & .. & .. & .. & .. & 18.8 & .. & 18.5 \\
\hline & $30-34$ & .. & .. & .. & .. & .. & 7.9 & .. & 11.4 \\
\hline & $35-39$ & .. & .. & .. & .. & .. & 3.5 & .. & 4.4 \\
\hline & $40+$ & .. & .. & .. & .. & .. & 17.6 & .. & 39.1 \\
\hline \multirow[t]{5}{*}{ Russian Federation } & $1-19$ & .. & .. & .. & 5.5 & 28.0 & 24.1 & 21.6 & 19.5 \\
\hline & $20-29$ & .. & .. & .. & 4.0 & 16.4 & 9.7 & 7.9 & 8.0 \\
\hline & $30-34$ & .. & .. & .. & .. & .. & .. & .. & .. \\
\hline & $35-39$ & .. & .. & .. & 89.1 & 43.4 & 65.9 & 70.4 & 72.2 \\
\hline & $40+$ & .. & .. & .. & 1.3 & 12.3 & 0.3 & 0.1 & 0.3 \\
\hline \multirow[t]{5}{*}{ South Africa } & $1-19$ & .. & .. & .. & .. & .. & .. & .. & 21.3 \\
\hline & $20-29$ & .. & .. &.. & .. & .. & .. & .. & 10.0 \\
\hline & $30-34$ & .. & .. & .. & .. & .. & .. & .. & 3.8 \\
\hline & $35-39$ &.. & .. & .. & .. & .. & .. & .. & 6.3 \\
\hline & $40+$ & .. & .. & .. & .. & .. & .. & .. & 58.8 \\
\hline
\end{tabular}




\begin{tabular}{llrrrrrrrr}
\hline Country & Hour bands & \multicolumn{1}{c}{ Year } & \multicolumn{1}{c}{} \\
\cline { 3 - 9 } & & 1980 & 1985 & 1990 & 1995 & 2000 & 2005 & 2010 & 2015 \\
\hline Colombia & $1-19$ &. &.. &.. &.. &.. & 27.4 & 31.0 & 29.3 \\
& $20-29$ &.. &.. &.. &.. &.. & 15.4 & 20.5 & 22.7 \\
& $30-34$ &.. &.. &.. &.. &.. & 9.8 & 5.6 & 6.6 \\
& $35-39$ &.. &.. &.. &.. &.. & 5.6 & 5.1 & 5.3 \\
& $40+$ &.. &.. &.. &.. &.. & 41.8 & 37.8 & 36.2 \\
\hline
\end{tabular}

Source: OECD.Stat.

Turning to women aged 65 and over, Table 1.6 demonstrates that even for this age group many women appear to maintain a strong connection with the paid labour force in terms of working hours. However, also apparent is that many are working substantially fewer hours than those in the 55-64 age group. To illustrate, in Australia in 2015, 19 per cent of women in the 55-64 age group worked between 1 and 19 hours per week compared with 43 per cent of women in the 65 or over age group. 
Table $1.7 \quad$ Incidence of temporary employment as a percentage of dependent employment for women aged 55-64 for selected countries

\begin{tabular}{|c|c|c|c|c|c|c|c|c|}
\hline \multirow[t]{2}{*}{ Country } & \multicolumn{8}{|c|}{ Year } \\
\hline & 1980 & 1985 & 1990 & 1995 & 2000 & 2005 & 2010 & 2015 \\
\hline Australia &.. &.. &.. & .. & .. & .. & 5.1 & 5.5 \\
\hline Austria &.. &.. &.. & 3.2 & 3.7 & 3.3 & 2.8 & 2.7 \\
\hline Belgium &.. & 3.0 & 2.1 & 2.2 & 6.1 & 7.0 & 4.2 & 4.0 \\
\hline Canada &.. &.. &.. &.. & 9.3 & 10.4 & 9.7 & 8.6 \\
\hline Chile &.. &.. &.. &.. &.. &.. & 18.8 & 20.4 \\
\hline Czech Republic & .. &.. &.. & 66.3 & 38.2 & 19.7 & 15.5 & 8.5 \\
\hline Denmark &.. & 4.4 & 6.9 & 4.3 & 5.4 & 5.5 & 3.6 & 4.0 \\
\hline Estonia &.. &.. &.. &.. & 1.1 & 0.9 & 1.1 & 1.5 \\
\hline Finland &.. & .. &.. &.. & 7.1 & 6.8 & 7.9 & 8.1 \\
\hline France &.. & 1.0 & 3.7 & 4.8 & 6.0 & 6.2 & 8.9 & 9.4 \\
\hline Germany & .. & 3.5 & 4.4 & 4.8 & 4.2 & 4.2 & 4.5 & 3.6 \\
\hline Greece &.. & 28.0 & 21.3 & 11.5 & 10.1 & 13.2 & 11.0 & 11.9 \\
\hline Hungary &.. &.. &.. &.. & 8.9 & 4.3 & 6.6 & 11.0 \\
\hline Iceland &.. &.. & .. & 4.5 & 10.3 & 5.3 & 4.9 & 4.3 \\
\hline Ireland &.. & 6.7 & 7.7 & 7.7 & 4.9 & 2.6 & 6.5 & 4.2 \\
\hline Italy &.. & 8.7 & 5.6 & 4.4 & 6.2 & 6.4 & 5.4 & 5.2 \\
\hline Japan & 22.6 & 22.7 & 22.5 & 21.5 & 22.2 & 24.3 & 23.1 & .. \\
\hline Korea & .. &.. & .. &.. & .. & 46.2 & 39.5 & 33.4 \\
\hline Latvia &.. &.. &.. &.. & 4.2 & 4.7 & 4.8 & 3.1 \\
\hline Lithuania &.. &.. &.. &.. & 1.5 & 1.4 & 0.8 & 1.5 \\
\hline Luxembourg &.. & 3.6 & 2.1 &.. & 1.2 & 3.6 & 2.5 & 4.2 \\
\hline Mexico &.. &.. &.. & 11.7 & 7.3 &.. &.. & .. \\
\hline Netherlands & .. & 4.1 & 4.4 & 8.9 & 9.1 & 8.0 & 7.5 & 6.2 \\
\hline Norway &.. &.. &.. &.. & 4.0 & 3.4 & 3.0 & 1.7 \\
\hline Poland &.. &.. & .. &.. &.. & 13.7 & 23.2 & 14.4 \\
\hline Portugal &.. &.. & 12.1 & 1.6 & 13.4 & 10.5 & 10.1 & 9.4 \\
\hline Slovak Republic &.. &.. & .. & 36.0 & 29.7 & 20.8 & 6.2 & 7.7 \\
\hline Slovenia &.. & .. & .. &.. & 23.0 & 17.0 & 10.3 & 8.5 \\
\hline Spain &.. &.. & 12.2 & 14.8 & 15.4 & 16.5 & 11.4 & 10.2 \\
\hline Sweden & .. &.. & .. &.. & 7.0 & 6.2 & 6.1 & 6.9 \\
\hline Switzerland &.. &.. & .. &.. & 4.5 & 4.7 & 4.2 & 4.5 \\
\hline Turkey &.. &.. & 50.0 & 51.1 & 54.6 & 30.0 & 30.6 & 15.9 \\
\hline United Kingdom & .. & 4.8 & 5.0 & 5.8 & 6.7 & 5.1 & 5.4 & 5.0 \\
\hline United States & .. &.. &.. & 3.0 &.. & 3.5 & .. & .. \\
\hline OECD countries & 8.5 & 8.9 & 9.5 & 9.9 & 10.0 & 9.5 & 9.3 & 8.6 \\
\hline Costa Rica &.. &.. & .. &.. &.. & .. & 5.1 & 4.6 \\
\hline
\end{tabular}




\begin{tabular}{|c|c|c|c|c|c|c|c|c|}
\hline \multirow[t]{2}{*}{ Country } & \multicolumn{8}{|c|}{ Year } \\
\hline & 1980 & 1985 & 1990 & 1995 & 2000 & 2005 & 2010 & 2015 \\
\hline European Union 28 & .. & 6.9 & 7.5 & 7.7 & 7.5 & 6.8 & 7.1 & 6.5 \\
\hline Russian Federation & .. & .. & .. & .. & 4.6 & 8.3 & 6.1 & 6.9 \\
\hline Colombia & .. & .. & .. &.. &.. & 12.9 & 14.3 & 19.5 \\
\hline
\end{tabular}

Source: OECD.Stat.

Table 1.7 shows the incidence of temporary employment (defined as wage and salary workers whose job has a pre-determined termination date) as a percentage of dependent employment for women in the 55-64 age range for selected countries over time. What is apparent from the figures for the OECD countries as a whole is that only approximately a tenth of women's employment can be defined this way. Thus, substantial majorities of such women's employment in most cases is defined as permanent. Relatively high levels are observed for a few countries (e.g. Chile and Korea). Also, notable once again are divergent trends for some countries, for instance in the cases of France and Greece. 
Table $1.8 \quad$ Incidence of temporary employment as a percentage of dependent employment for women 65 and over for selected countries

\begin{tabular}{|c|c|c|c|c|c|c|c|c|}
\hline \multirow[t]{2}{*}{ Country } & \multicolumn{8}{|c|}{ Year } \\
\hline & 1980 & 1985 & 1990 & 1995 & 2000 & 2005 & 2010 & 2015 \\
\hline Australia &.. & .. & .. & .. & .. & .. & 47.7 & 37.4 \\
\hline Austria &.. & .. &.. & 9.1 & .. & 8.7 & 19.3 & 41.7 \\
\hline Belgium &.. & .. & 57.9 & 42.6 & 58.6 & 14.3 & 40.3 & 22.4 \\
\hline Canada &.. &.. &.. & .. & 32.1 & 45.9 & 36.9 & 44.2 \\
\hline Chile &.. & .. &.. & .. &.. & .. & 10.5 & 20.0 \\
\hline Czech Republic &.. & .. &.. & 43.3 & 41.8 & 43.9 & 48.4 & 51.9 \\
\hline Denmark & .. & 29.9 & 31.8 & .. & 39.6 & 34.9 & 22.3 & 24.5 \\
\hline Estonia &.. & .. &.. & .. & 72.2 & 57.2 & 100.0 & 72.5 \\
\hline Finland & .. & .. &.. & .. &.. & 43.8 & 39.5 & 59.3 \\
\hline France & .. & 100.0 & 72.4 & 25.0 & 12.6 & 45.3 & 40.1 & 45.7 \\
\hline Germany & .. & 44.8 & 42.5 & 44.0 & 24.7 & 42.3 & 38.3 & 38.1 \\
\hline Greece & .. & 46.0 & 34.4 & 24.0 & 45.9 & 32.6 & 75.9 & 54.5 \\
\hline Hungary & .. &.. &.. & .. & 46.7 & 24.0 & 46.1 & 51.7 \\
\hline Iceland & .. & .. &.. & 39.5 & 27.0 & 51.8 & 50.6 & 29.7 \\
\hline Ireland & .. & 23.3 & 30.1 & 38.6 & 41.7 & 33.2 & 64.5 & 74.1 \\
\hline Italy &.. & 40.2 & 42.7 & 49.7 & 35.0 & 17.0 & 23.6 & 30.6 \\
\hline Japan & 38.9 & 41.2 & 37.5 & 33.3 & 30.1 & 34.5 & 37.7 & .. \\
\hline Korea &.. & .. &.. & .. & .. & 54.4 & 49.9 & 50.6 \\
\hline Latvia & .. & .. & .. &.. & 43.4 & 16.0 & 53.1 & 46.2 \\
\hline Lithuania & .. & .. & .. & .. & 100.0 & 81.8 & 31.7 & 48.3 \\
\hline Luxembourg &.. & 100.0 &.. &.. & 100.0 & .. &.. & .. \\
\hline Mexico & .. & .. & .. & 2.2 & 7.6 & .. &.. & .. \\
\hline Netherlands & .. & 19.9 & .. & 25.6 & 24.3 & 16.6 & 21.6 & 24.4 \\
\hline Norway & .. &.. & .. &.. & .. & 59.2 & 36.6 & 57.3 \\
\hline Poland & .. & .. &.. &.. & .. & 34.9 & 32.4 & 36.3 \\
\hline Portugal & .. &.. & 28.0 & 12.6 & 29.4 & 37.4 & 44.7 & 46.0 \\
\hline Slovak & .. & .. & .. & 41.2 & 19.1 & 57.9 & 41.9 & 40.7 \\
\hline \multicolumn{9}{|l|}{ Republic } \\
\hline Slovenia & .. & .. & .. & .. & 19.9 & 40.3 & 45.0 & 45.5 \\
\hline Spain &.. &.. & 49.1 & 56.5 & 56.7 & 51.7 & 54.6 & 67.4 \\
\hline Sweden &.. &.. &.. &.. &.. & .. & 46.0 & 49.4 \\
\hline Switzerland & .. & .. & .. & .. & 16.3 & 39.3 & 30.3 & 29.8 \\
\hline Turkey &.. & .. & 15.4 & 24.4 & 25.0 & 28.6 & 45.5 & 18.2 \\
\hline United &.. & 36.2 & 38.5 & 39.2 & 41.1 & 43.2 & 43.6 & 44.9 \\
\hline \multicolumn{9}{|l|}{ Kingdom } \\
\hline United States &.. & .. & .. & 47.3 & .. & 64.8 & .. &.. \\
\hline
\end{tabular}




\begin{tabular}{|c|c|c|c|c|c|c|c|c|}
\hline \multirow[t]{2}{*}{ Country } & \multicolumn{8}{|c|}{ Year } \\
\hline & 1980 & 1985 & 1990 & 1995 & 2000 & 2005 & 2010 & 2015 \\
\hline $\begin{array}{l}\text { OECD } \\
\text { countries }\end{array}$ & 39.8 & 40.6 & 39.6 & 38.1 & 33.5 & 41.6 & 42.5 & 43.6 \\
\hline Costa Rica & .. & .. & .. & .. & .. & .. & 14.5 & 4.5 \\
\hline $\begin{array}{l}\text { European } \\
\text { Union } 28\end{array}$ & .. & 38.3 & 38.3 & 39.8 & 36.9 & 38.2 & 40.4 & 42.8 \\
\hline $\begin{array}{l}\text { Russian } \\
\text { Federation }\end{array}$ & .. & .. & .. & .. & 43.3 & 49.9 & 50.0 & 53.3 \\
\hline Colombia & .. & .. & .. & .. & .. & 29.6 & 18.2 & 24.6 \\
\hline
\end{tabular}

Source: OECD.Stat.

Table 1.8 shows the incidence of temporary employment (defined as wage and salary workers whose job has a pre-determined termination date) as a percentage of dependent employment for women aged 65 or over for selected countries over time. For such women the incidence of temporary employment is considerably higher than for those in the 55-64 age group. Even so, for many countries it is not the norm. Across the OECD countries approximately two-fifths of women in this age group have temporary contracts. Altogether, Tables 1.7 and 1.8 point to a clear transition in terms of the nature of employment contracts for women after the age of 65 . Nevertheless, they again demonstrate heterogeneity in terms of older women's work. 
Table 1.9 Incidence of involuntary part-time working for women aged 55-64

\begin{tabular}{|c|c|c|c|c|c|c|c|c|c|}
\hline \multirow[t]{2}{*}{ Country } & \multirow{2}{*}{$\begin{array}{l}\text { Incidence of } \\
\text { involuntary } \\
\text { part time } \\
\text { workers }\end{array}$} & \multicolumn{8}{|c|}{ Year } \\
\hline & & 1980 & 1985 & 1990 & 1995 & 2000 & 2005 & 2010 & 2015 \\
\hline \multirow[t]{3}{*}{ Australia } & $\begin{array}{l}\text { A. Share of } \\
\text { involuntary } \\
\text { part-timers }\end{array}$ &.. &.. &.. & 36.4 & 47.3 & 53.9 & 53.6 & 60.4 \\
\hline & $\begin{array}{l}\text { B. Share of } \\
\text { involuntary } \\
\text { part-timers } \\
\text { in total } \\
\text { employment }\end{array}$ &.. &.. &.. & 4.8 & 5.8 & 6.3 & 6.5 & 8.4 \\
\hline & $\begin{array}{l}\text { C. Share of } \\
\text { involuntary } \\
\text { part-timers as } \\
\% \text { of part-time } \\
\text { employment }\end{array}$ &.. &.. &.. & 9.6 & 11.3 & 12.5 & 13.3 & 16.8 \\
\hline \multirow[t]{3}{*}{ Austria } & A &.. &.. &.. & 95.5 & 48.2 & 67.7 & 68.9 & 73.6 \\
\hline & B &.. &.. &.. & 2.8 & 3.5 & 6.0 & 4.4 & 5.6 \\
\hline & $\mathrm{C}$ &.. &.. & .. & 7.3 & 9.4 & 12.7 & 9.0 & 11.0 \\
\hline \multirow[t]{3}{*}{ Belgium } & A &.. & 64.7 & 91.3 & 61.5 & 82.4 & 71.8 & 74.6 & 75.0 \\
\hline & B &.. & 1.7 & 3.8 & 2.2 & 4.6 & 4.7 & 2.7 & 2.4 \\
\hline & $\mathrm{C}$ &.. & 8.1 & 14.8 & 7.7 & 10.1 & 10.2 & 5.1 & 4.7 \\
\hline \multirow[t]{3}{*}{ Canada } & A & 65.6 & 66.8 & 65.1 & 63.5 & 57.0 & 63.0 & 58.4 & 61.1 \\
\hline & B & 3.1 & 5.8 & 5.1 & 8.7 & 5.8 & 6.4 & 6.8 & 6.6 \\
\hline & $\mathrm{C}$ & 11.2 & 19.7 & 16.4 & 26.6 & 19.3 & 21.8 & 23.9 & 25.9 \\
\hline \multirow[t]{3}{*}{ Chile } & A &.. &.. &.. & .. &.. &.. & 48.6 & 55.6 \\
\hline & B &.. &.. &.. &.. &.. &.. & 16.9 & 13.6 \\
\hline & $\mathrm{C}$ &.. &.. &.. &.. &.. &.. & 51.2 & 43.6 \\
\hline Czech & A &.. &.. &.. & 75.8 & 87.2 & 63.1 & 50.0 & 71.7 \\
\hline \multirow[t]{2}{*}{ Republic } & B &.. &.. &.. & 8.1 & 6.0 & 1.1 & 1.3 & 1.4 \\
\hline & $\mathrm{C}$ &.. &.. &.. & 22.0 & 21.9 & 7.1 & 9.1 & 12.0 \\
\hline \multirow[t]{3}{*}{ Denmark } & A &.. & 84.2 & 77.5 & 70.9 & 83.7 & 76.6 & 73.0 & 83.8 \\
\hline & B &.. & 4.3 & 5.0 & 8.5 & 6.3 & 5.2 & 5.6 & 6.0 \\
\hline & $\mathrm{C}$ &.. & 7.2 & 10.2 & 19.1 & 15.5 & 16.1 & 14.0 & 19.0 \\
\hline \multirow[t]{3}{*}{ Estonia } & A &.. &.. &.. &.. &.. & 81.8 & 69.2 & 76.5 \\
\hline & B &.. &.. &.. &.. &.. & 1.9 & 3.6 & 2.1 \\
\hline & C &.. & .. &.. &.. &.. & 17.2 & 23.5 & 17.2 \\
\hline \multirow[t]{3}{*}{ Finland } & A &.. &.. &.. &.. & 83.3 & 73.7 & 73.5 & 73.5 \\
\hline & B &.. &.. &.. &.. & 4.3 & 3.0 & 3.2 & 3.7 \\
\hline & $\mathrm{C}$ &.. &.. &.. &.. & 19.2 & 13.8 & 13.7 & 20.6 \\
\hline
\end{tabular}




\begin{tabular}{|c|c|c|c|c|c|c|c|c|c|}
\hline \multirow[t]{2}{*}{ Country } & \multirow{2}{*}{$\begin{array}{l}\text { Incidence of } \\
\text { involuntary } \\
\text { part time } \\
\text { workers }\end{array}$} & \multicolumn{8}{|c|}{ Year } \\
\hline & & 1980 & 1985 & 1990 & 1995 & 2000 & 2005 & 2010 & 2015 \\
\hline \multirow[t]{3}{*}{ France } & A & .. &.. &.. & 74.2 & 64.3 & 78.8 & 81.1 & 80.5 \\
\hline & B &.. &.. &.. & 4.4 & 2.7 & 8.9 & 9.1 & 12.9 \\
\hline & $\mathrm{C}$ & .. & .. &.. & 12.6 & 7.5 & 26.7 & 27.3 & 37.7 \\
\hline \multirow[t]{3}{*}{ Germany } & A & .. & 87.7 & 87.1 & 77.9 & 76.2 & 74.9 & 72.3 & 76.4 \\
\hline & B &.. & 1.5 & 1.6 & 3.6 & 4.7 & 8.0 & 10.4 & 7.4 \\
\hline & $\mathrm{C}$ &.. & 3.9 & 3.6 & 7.8 & 9.8 & 15.9 & 20.8 & 14.3 \\
\hline \multirow[t]{3}{*}{ Greece } & A &.. & 28.6 & 44.4 & 50.0 & 47.5 & 56.3 & 48.3 & 59.0 \\
\hline & B &.. & 0.8 & 1.0 & 1.9 & 1.3 & 2.2 & 2.2 & 6.8 \\
\hline & $\mathrm{C}$ & .. & 7.5 & 10.7 & 15.0 & 12.9 & 20.1 & 18.5 & 52.0 \\
\hline \multirow[t]{3}{*}{ Hungary } & $\mathrm{A}$ & 61.5 & 61.5 & 61.5 & 61.5 & 44.0 & 72.2 & 61.4 & 58.3 \\
\hline & B & .. & .. & .. & 4.8 & 1.4 & 1.5 & 1.6 & 2.4 \\
\hline & $\mathrm{C}$ &.. & .. &.. & 15.8 & 6.8 & 11.6 & 11.6 & 16.2 \\
\hline \multirow[t]{3}{*}{ Iceland } & A & .. & .. &.. &.. & 100.0 &.. & 82.4 & 92.9 \\
\hline & $\mathrm{B}$ &.. &.. &.. &.. & 4.9 &.. & 11.0 & 8.5 \\
\hline & $\mathrm{C}$ &.. &.. &.. & .. & 10.7 &.. & 28.6 & 28.4 \\
\hline \multirow[t]{3}{*}{ Ireland } & A &.. & 44.4 & 31.6 & 41.2 & 38.2 &.. & 54.9 & 49.4 \\
\hline & B & .. & 1.7 & 2.6 & 5.1 & 4.8 &.. & 9.4 & 9.9 \\
\hline & $\mathrm{C}$ &.. & 6.3 & 9.8 & 15.5 & 10.7 &.. & 19.9 & 23.0 \\
\hline \multirow[t]{3}{*}{ Israel } & A &.. &.. &.. & 71.7 & 71.7 & 79.8 & 80.6 & 76.1 \\
\hline & B &.. & .. & .. & 5.6 & 5.8 & 9.4 & 6.3 & 4.2 \\
\hline & $\mathrm{C}$ &.. &.. &.. & 11.8 & 12.4 & 21.8 & 15.0 & 11.8 \\
\hline \multirow[t]{3}{*}{ Italy } & A & .. & 30.4 & 43.5 & 44.2 & 41.8 & 60.8 & 67.9 & 67.4 \\
\hline & B &.. & 1.7 & 2.5 & 2.1 & 2.8 & 5.0 & 8.4 & 13.0 \\
\hline & $\mathrm{C}$ &.. & 12.2 & 23.8 & 17.2 & 20.6 & 26.7 & 40.5 & 54.7 \\
\hline \multirow[t]{3}{*}{ Japan } & A &.. &.. &.. &.. &.. & 57.9 & 53.2 & 55.3 \\
\hline & B &.. &.. &.. & .. & .. & 7.2 & 8.5 & 5.6 \\
\hline & $\mathrm{C}$ &.. &.. & .. &.. &.. & 19.4 & 21.0 & 13.3 \\
\hline \multirow[t]{3}{*}{ Latvia } & A &.. & .. &.. & .. & .. & 64.0 & 60.4 & 69.2 \\
\hline & B &.. &.. &.. &.. &.. & 4.9 & 4.3 & 4.1 \\
\hline & $\mathrm{C}$ &.. &.. &.. & .. &.. & 35.9 & 36.4 & 36.4 \\
\hline \multirow[t]{3}{*}{ Lithuania } & A &.. &.. &.. &.. &.. & 48.2 & 54.8 & 68.1 \\
\hline & B &.. &.. &.. &.. &.. & 4.8 & 3.8 & 5.1 \\
\hline & $\mathrm{C}$ &.. &.. &.. &.. &.. & 35.7 & 30.9 & 37.7 \\
\hline \multirow[t]{3}{*}{ Luxembourg } & A &.. &.. & 100.0 & 100.0 &.. & 100.0 & 100.0 & 85.7 \\
\hline & B &.. & 0.0 & 3.3 & 3.5 & 0.0 & 3.5 & 2.5 & 5.8 \\
\hline & $\mathrm{C}$ & .. & 0.0 & 16.5 & 14.4 & 0.0 & 7.4 & 6.2 & 12.4 \\
\hline
\end{tabular}




\begin{tabular}{|c|c|c|c|c|c|c|c|c|c|}
\hline \multirow[t]{2}{*}{ Country } & \multirow{2}{*}{$\begin{array}{l}\text { Incidence of } \\
\text { involuntary } \\
\text { part time } \\
\text { workers }\end{array}$} & \multicolumn{8}{|c|}{ Year } \\
\hline & & 1980 & 1985 & 1990 & 1995 & 2000 & 2005 & 2010 & 2015 \\
\hline \multirow[t]{3}{*}{ Netherlands } & A & .. & 77.2 & 74.2 & 59.4 & 58.2 & 43.6 & 59.3 & 62.6 \\
\hline & B &.. & 4.9 & 14.7 & 1.5 & 1.6 & 1.1 & 3.0 & 5.4 \\
\hline & $\mathrm{C}$ & .. & 7.1 & 18.8 & 1.8 & 2.0 & 1.3 & 3.7 & 6.9 \\
\hline \multirow[t]{3}{*}{ New Zealand } & A & .. &.. & 55.6 & 52.5 & 66.0 & 70.4 & 61.0 & 71.8 \\
\hline & B &.. &.. & 4.8 & 5.6 & 7.9 & 3.9 & 4.4 & 4.6 \\
\hline & $\mathrm{C}$ & .. & .. & 10.0 & 12.9 & 18.9 & 10.3 & 12.6 & 14.5 \\
\hline \multirow[t]{3}{*}{ Norway } & A &.. &.. & 80.0 & 85.7 & 56.5 & 67.8 & 54.9 & 76.5 \\
\hline & B & .. & .. & 4.0 & 4.5 & 1.0 & 2.3 & 0.6 & 1.0 \\
\hline & $\mathrm{C}$ & .. & .. & 6.8 & 8.0 & 2.0 & 5.0 & 1.3 & 2.3 \\
\hline \multirow[t]{3}{*}{ Poland } & A & .. & .. & .. &.. &.. & 29.6 & 40.7 & 53.7 \\
\hline & B & .. & .. & .. &.. & .. & 1.2 & 1.4 & 2.1 \\
\hline & $\mathrm{C}$ & .. & .. & .. &.. & .. & 4.1 & 5.0 & 14.3 \\
\hline \multirow[t]{3}{*}{ Portugal } & A & .. & .. & 64.6 & 80.9 & 78.2 & 77.5 & 77.2 & 71.4 \\
\hline & B &.. &.. & 2.5 & 4.2 & 6.2 & 5.7 & 5.3 & 7.9 \\
\hline & $\mathrm{C}$ & .. & .. & 14.6 & 20.2 & 18.6 & 18.9 & 20.7 & 38.5 \\
\hline Slovak & A & .. & .. & .. & 56.2 & 73.4 & 94.0 & 45.9 & 57.4 \\
\hline \multirow[t]{2}{*}{ Republic } & B &.. &.. &.. & 4.7 & 2.5 & 5.5 & 3.1 & 4.5 \\
\hline & $\mathrm{C}$ &.. &.. &.. & 26.3 & 15.0 & 31.9 & 33.7 & 45.3 \\
\hline \multirow[t]{3}{*}{ Slovenia } & A &.. &.. &.. &.. &.. &.. & 40.0 & 60.0 \\
\hline & B &.. &.. &.. &.. &.. &.. & 0.6 & 0.7 \\
\hline & $\mathrm{C}$ & .. & .. &.. &.. &.. &.. & 2.3 & 4.0 \\
\hline \multirow[t]{3}{*}{ Spain } & A & .. & .. & 84.3 & 87.2 & 84.4 & 81.9 & 80.6 & 80.4 \\
\hline & B &.. &.. & 2.1 & 2.2 & 1.8 & 6.3 & 9.5 & 12.7 \\
\hline & $\mathrm{C}$ &.. &.. & 13.6 & 13.1 & 9.8 & 24.3 & 43.7 & 58.0 \\
\hline \multirow[t]{3}{*}{ Sweden } & A & 87.3 & 87.3 & 82.0 & 77.7 & 75.7 & 66.1 & 67.6 & 54.4 \\
\hline & B & 2.9 & 2.9 & 2.9 & 6.0 & 3.3 & 2.2 & 2.5 & 2.0 \\
\hline & $\mathrm{C}$ & 5.3 & 5.5 & 5.6 & 11.9 & 8.5 & 6.0 & 6.7 & 6.4 \\
\hline \multirow[t]{3}{*}{ Switzerland } & A &.. & .. &.. & .. & .. &.. &.. &.. \\
\hline & B &.. &.. &.. &.. &.. &.. &.. &.. \\
\hline & $\mathrm{C}$ &.. &.. &.. &.. &.. &.. &.. &.. \\
\hline \multirow[t]{3}{*}{ Turkey } & A & .. &.. &.. & .. & .. &.. & 21.8 & 19.9 \\
\hline & B &.. & .. &.. &.. &.. &.. & 0.6 & 0.6 \\
\hline & $\mathrm{C}$ &.. &.. &.. &.. &. &.. & 1.6 & 2.1 \\
\hline United & A &.. & 65.4 & 50.2 & 49.6 & 52.6 & 54.9 & 44.9 & 61.6 \\
\hline \multirow[t]{2}{*}{ Kingdom } & B &.. & 3.4 & 2.4 & 4.5 & 3.4 & 2.5 & 3.2 & 5.0 \\
\hline & $\mathrm{C}$ & .. & 5.7 & 4.1 & 7.6 & 6.0 & 4.6 & 6.2 & 10.2 \\
\hline
\end{tabular}




\begin{tabular}{|c|c|c|c|c|c|c|c|c|c|}
\hline \multirow[t]{2}{*}{ Country } & \multirow{2}{*}{$\begin{array}{l}\text { Incidence of } \\
\text { involuntary } \\
\text { part time } \\
\text { workers }\end{array}$} & \multicolumn{8}{|c|}{ Year } \\
\hline & & 1980 & 1985 & 1990 & 1995 & 2000 & 2005 & 2010 & 2015 \\
\hline \multirow[t]{3}{*}{ United States } & A &.. &.. &.. & .. & 63.2 & 60.6 & 64.5 & 62.0 \\
\hline & B &.. &.. &.. &.. & 0.6 & 0.7 & 1.4 & 1.2 \\
\hline & $\mathrm{C}$ & .. & .. & .. & .. & 2.3 & 3.0 & 5.6 & 5.4 \\
\hline \multirow{3}{*}{$\begin{array}{l}\text { OECD } \\
\text { countries }\end{array}$} & A &.. & 62.7 & 62.8 & 60.7 & 60.5 & 62.7 & 61.2 & 66.1 \\
\hline & B & .. & 3.0 & 2.6 & 3.1 & 2.7 & 3.9 & 4.7 & 4.8 \\
\hline & $\mathrm{C}$ & .. & 9.2 & 8.1 & 8.8 & 7.9 & 11.6 & 13.8 & 14.4 \\
\hline European & A &.. & 64.2 & 63.6 & 63.2 & 63.0 & 70.4 & 68.8 & 72.1 \\
\hline \multirow[t]{2}{*}{ Union 22} & B & .. & 3.4 & 3.1 & 3.6 & 3.5 & 5.0 & 6.4 & 7.7 \\
\hline & $\mathrm{C}$ & .. & 8.9 & 8.3 & 9.2 & 8.8 & 12.9 & 16.8 & 20.7 \\
\hline European & $\mathrm{A}$ & .. & 64.2 & 63.6 & 63.2 & 63.0 & 68.6 & 67.3 & 70.9 \\
\hline \multirow[t]{2}{*}{ Union 28} & $\mathrm{~B}$ & .. & 3.4 & 3.1 & 3.6 & 3.3 & 5.0 & 6.3 & 7.6 \\
\hline & $\mathrm{C}$ & .. & 8.9 & 8.3 & 9.2 & 8.2 & 13.0 & 16.8 & 20.8 \\
\hline \multirow[t]{3}{*}{ G7 countries } & A & .. & 62.3 & 61.6 & 60.4 & 62.7 & 63.5 & 61.4 & 66.5 \\
\hline & $\mathrm{B}$ &.. &.. &.. &.. &.. &.. &.. &.. \\
\hline & $\mathrm{C}$ &.. &.. &.. &.. &.. &.. &.. &.. \\
\hline \multirow[t]{3}{*}{ Bulgaria } & A &.. &.. & .. &.. &.. & 53.6 & 59.6 & 62.4 \\
\hline & B & .. & .. &.. &.. &.. & 3.0 & 1.5 & 2.0 \\
\hline & $\mathrm{C}$ & .. & .. &.. &.. &.. & 51.3 & 36.5 & 57.2 \\
\hline \multirow[t]{3}{*}{ Croatia } & A &.. &.. &.. &.. &.. & 30.0 & 35.7 & 17.6 \\
\hline & $\mathrm{B}$ &.. &.. &.. & .. &.. & 0.9 & 1.2 & 0.3 \\
\hline & $\mathrm{C}$ &.. &.. & .. &.. &.. & 2.9 & 6.3 & 2.8 \\
\hline \multirow[t]{3}{*}{ Cyprus } & A &.. & .. & .. &.. &.. & 50.0 & 50.0 & 44.7 \\
\hline & B &.. &.. &.. &.. &.. & 4.1 & 2.0 & 11.4 \\
\hline & $\mathrm{C}$ &.. &.. &.. &.. &.. & 17.5 & 12.7 & 52.9 \\
\hline FYR of & A &.. &.. & .. & .. &.. &.. & 31.3 & 27.3 \\
\hline \multirow[t]{2}{*}{ Macedonia } & B &.. &.. &.. &.. &.. &.. & 1.9 & 0.8 \\
\hline & C &.. & .. & .. &.. &.. &.. & 17.4 & 16.0 \\
\hline \multirow[t]{3}{*}{ Malta } & A &.. & .. &.. & .. & .. & 33.3 & 33.3 & 50.0 \\
\hline & B &.. &.. & .. & .. &.. & 3.1 & 2.4 & 4.7 \\
\hline & $\mathrm{C}$ &.. &.. & .. &.. &.. & 13.0 & 6.1 & 13.5 \\
\hline \multirow[t]{3}{*}{ Romania } & A &.. &.. &.. &.. &.. & 40.0 & 32.5 & 35.3 \\
\hline & B &.. &.. & .. &.. &.. & 2.9 & 2.7 & 3.5 \\
\hline & $\mathrm{C}$ &.. &.. &.. &.. &.. & 14.5 & 12.8 & 18.0 \\
\hline Russian & A &.. &.. &.. & 31.9 & 43.7 & 47.0 & 64.5 & 63.7 \\
\hline \multirow[t]{2}{*}{ Federation } & B &.. & .. & .. & 1.8 & 0.4 & 0.3 & 0.9 & 0.6 \\
\hline & $\mathrm{C}$ & .. &.. &.. & 13.4 & 1.7 & 2.2 & 7.4 & 4.7 \\
\hline
\end{tabular}




\begin{tabular}{|c|c|c|c|c|c|c|c|c|c|}
\hline \multirow[t]{2}{*}{ Country } & \multirow{2}{*}{$\begin{array}{l}\text { Incidence of } \\
\text { involuntary } \\
\text { part time } \\
\text { workers }\end{array}$} & \multicolumn{8}{|c|}{ Year } \\
\hline & & 1980 & 1985 & 1990 & 1995 & 2000 & 2005 & 2010 & 2015 \\
\hline \multirow[t]{3}{*}{ Colombia } & A & .. & .. & .. & .. &.. & 52.1 & 56.0 & 55.2 \\
\hline & B & .. & .. & .. & .. & .. & 12.2 & 11.4 & 8.8 \\
\hline & $\mathrm{C}$ &.. & .. & .. & .. & .. & 34.6 & 32.5 & 25.8 \\
\hline
\end{tabular}

Source: OECD.Stat.

Another consideration when exploring issues of flexible working is the extent of under-employment. Table 1.9 shows the incidence of involuntary part-time working among women in the 55-64 age group. From these figures it can be seen that women are more likely to be under-employed, and in a number of cases, are substantially under-employed. Thus, for the OECD countries as a whole, approximately two-thirds of women in the 55-64 age group were in involuntary part-time employment in 2015. However, according to Table 1.9 this has not always been the case. So, for instance, in Australia up to the year 2000, men were more likely to experience under-employment. Similarly, in the case of Italy, up to the year 2000 men outnumbered women in terms of involuntary part-time employment. Elsewhere, for instance in Belgium and Denmark, women have heavily outnumbered men.

Table 1.9 also shows the incidence of involuntary part-time working as a proportion of total employment. Here, for a number of countries there has been a modest upward trend. However, for the OECD countries as a whole, despite an upward trend, involuntary part-time employment remains relatively rare.

In addition, Table 1.9 shows the incidence of involuntary part-time employment as a percentage of part-time employment. Here, it is apparent that such workers represent a minority of part-time workers overall, although a growing proportion across the OECD countries. Also, for a number of countries, such workers represent a large minority or even a majority of part-time workers, for instance in Canada, Chile, Finland, Greece, Iceland, Ireland, Italy, Latvia, Lithuania, Portugal and the Slovak Republic. Thus, a lack of access to adequate work appears to be a serious problem for many older women in part-time roles in a number of countries. This contrasts with the much-promoted notion that older workers would benefit from having greater access to bridge jobs in the lead up to retirement in the form of part-time work. 
Table $1.10 \quad$ Incidence of involuntary part-time working for women aged 65 and over

\begin{tabular}{|c|c|c|c|c|c|c|c|c|c|}
\hline \multirow[t]{2}{*}{ Country } & \multirow[t]{2}{*}{ Series } & \multicolumn{8}{|c|}{ Year } \\
\hline & & 1980 & 1985 & 1990 & 1995 & 2000 & 2005 & 2010 & 2015 \\
\hline \multirow[t]{3}{*}{ Australia } & $\begin{array}{l}\text { A. Share of } \\
\text { involuntary } \\
\text { part-timers }\end{array}$ &.. &.. &.. & 3.7 & 26.4 & 27.0 & 39.6 & 38.9 \\
\hline & $\begin{array}{l}\text { B. Share of } \\
\text { involuntary } \\
\text { part-timers } \\
\text { in total } \\
\text { employment }\end{array}$ &.. &.. &.. & 0.2 & 1.9 & 3.2 & 4.3 & 5.2 \\
\hline & $\begin{array}{l}\text { C. Share of } \\
\text { involuntary } \\
\text { part-timers as } \\
\% \text { of part-time } \\
\text { employment }\end{array}$ &.. &.. &.. & 0.3 & 2.7 & 4.7 & 6.3 & 7.5 \\
\hline \multirow[t]{3}{*}{ Austria } & A &.. &.. &.. & 75.0 & 10.0 & 20.0 & 40.0 & 41.7 \\
\hline & B &.. &.. &.. & 1.7 & 0.8 & 0.8 & 0.7 & 1.6 \\
\hline & $\mathrm{C}$ &.. & .. &.. & 2.5 & 1.7 & 1.2 & 0.9 & 1.9 \\
\hline \multirow[t]{3}{*}{ Belgium } & A &.. & .. &.. & 100.0 &.. &.. & 12.5 & 83.3 \\
\hline & B &.. & .. &.. & 2.3 & .. &.. & 0.8 & 3.2 \\
\hline & $\mathrm{C}$ &.. & .. &.. & 9.8 & .. &.. & 1.1 & 5.3 \\
\hline \multirow[t]{3}{*}{ Canada } & A &.. & .. &.. &.. & .. &.. &.. &.. \\
\hline & B &.. &.. &.. &.. &.. &.. &.. &.. \\
\hline & $\mathrm{C}$ &.. &.. &.. &.. &.. &.. &.. &.. \\
\hline \multirow[t]{3}{*}{ Chile } & A &.. & .. &.. &.. & .. &.. & 36.8 & 42.1 \\
\hline & B &.. &.. &.. &.. &.. &.. & 17.4 & 17.7 \\
\hline & $\mathrm{C}$ &.. & .. &.. &.. &.. &.. & 36.9 & 32.5 \\
\hline Czech & A &.. &.. &.. & 34.6 & 46.7 & 83.0 & 39.7 & 42.2 \\
\hline \multirow[t]{2}{*}{ Republic } & B &.. &.. &.. & 7.2 & 11.8 & 2.8 & 1.2 & 0.5 \\
\hline & $\mathrm{C}$ &.. &.. &.. & 11.7 & 19.6 & 4.1 & 1.8 & 0.7 \\
\hline \multirow[t]{3}{*}{ Denmark } & A &.. & 50.0 & 46.2 & 50.0 & 48.0 & 42.1 & 31.6 & 40.0 \\
\hline & B &.. & 2.8 & 3.9 & 9.6 & 19.6 & 6.3 & 3.9 & 3.9 \\
\hline & $\mathrm{C}$ &.. & 3.8 & 5.2 & 12.9 & 25.1 & 8.7 & 5.5 & 5.9 \\
\hline \multirow[t]{3}{*}{ Estonia } & A &.. &.. &.. &.. &.. & 78.6 & 50.0 & 85.7 \\
\hline & B &.. &.. &.. &.. &.. & 8.7 & 3.5 & 3.6 \\
\hline & $\mathrm{C}$ &.. &.. &.. &.. &.. & 17.3 & 6.6 & 7.5 \\
\hline \multirow[t]{3}{*}{ Finland } & A &.. &.. &.. &.. & .. &.. &.. & .. \\
\hline & B &.. &.. &.. &.. &.. &.. &.. &.. \\
\hline & $\mathrm{C}$ &.. &.. &.. & .. &.. &.. &.. &.. \\
\hline
\end{tabular}




\begin{tabular}{|c|c|c|c|c|c|c|c|c|c|}
\hline \multirow[t]{2}{*}{ Country } & \multirow[t]{2}{*}{ Series } & \multicolumn{8}{|c|}{ Year } \\
\hline & & 1980 & 1985 & 1990 & 1995 & 2000 & 2005 & 2010 & 2015 \\
\hline \multirow[t]{3}{*}{ France } & A & .. & .. & .. & 55.0 & .. & 15.4 & 50.0 & 25.8 \\
\hline & B & .. &.. & .. & 1.8 &.. & 0.4 & 4.8 & 2.8 \\
\hline & $\mathrm{C}$ &.. &.. & .. & 4.4 & .. & 0.9 & 8.1 & 4.5 \\
\hline \multirow[t]{3}{*}{ Germany } & A & .. &.. & 50.0 & 46.2 & 25.0 & 42.6 & 71.2 & 50.0 \\
\hline & B & .. &.. & 0.3 & 0.5 & 0.4 & 1.2 & 2.3 & 1.0 \\
\hline & $\mathrm{C}$ & .. & .. & 0.5 & 0.8 & 0.6 & 1.5 & 2.8 & 1.1 \\
\hline \multirow[t]{3}{*}{ Greece } & A & .. & 25.0 & 33.3 & 50.0 & 46.7 & 40.0 & 50.0 & 21.7 \\
\hline & B & .. & 0.3 & 1.1 & 1.7 & 2.6 & 1.0 & 2.0 & 2.1 \\
\hline & $\mathrm{C}$ & .. & 0.9 & 3.2 & 6.4 & 10.4 & 3.5 & 7.9 & 9.9 \\
\hline \multirow[t]{3}{*}{ Hungary } & A & 50.0 & 50.0 & 50.0 & 50.0 & 100.0 &.. & 33.3 & 83.3 \\
\hline & B &.. & .. & .. & 4.4 & 3.0 & .. & 0.7 & 3.2 \\
\hline & $\mathrm{C}$ & .. & .. & .. & 6.7 & 6.2 & .. & 1.2 & 6.2 \\
\hline \multirow[t]{3}{*}{ Iceland } & A & .. & .. & .. & .. & 100.0 &.. & 100.0 & 66.7 \\
\hline & B &.. &.. &.. & .. & 5.0 &.. & 7.1 & 10.6 \\
\hline & $\mathrm{C}$ & .. &.. & .. & .. & 8.7 & .. & 14.3 & 16.7 \\
\hline \multirow[t]{3}{*}{ Ireland } & A & .. & .. & .. & .. & 25.0 & .. & 43.8 & 52.2 \\
\hline & B & .. & 0.0 & 0.0 & 0.0 & 1.6 & .. & 5.8 & 6.7 \\
\hline & $\mathrm{C}$ & .. & 0.0 & 0.0 & 0.0 & 3.5 & .. & 8.2 & 10.2 \\
\hline \multirow[t]{3}{*}{ Israel } & A & .. &.. &.. & 27.8 & 41.7 & 59.5 & 65.0 & 56.2 \\
\hline & B & .. &.. & .. & 0.8 & 1.8 & 4.1 & 4.0 & 2.3 \\
\hline & $\mathrm{C}$ & .. & .. & .. & 1.0 & 2.1 & 5.4 & 5.3 & 3.3 \\
\hline \multirow[t]{3}{*}{ Italy } & A & .. & 26.4 & 44.0 & 50.0 & 62.5 & 40.4 & 34.0 & 37.5 \\
\hline & B & .. & 1.5 & 2.1 & 1.4 & 0.6 & 3.3 & 5.6 & 9.6 \\
\hline & $\mathrm{C}$ & .. & 5.7 & 9.7 & 7.5 & 2.5 & 9.7 & 15.5 & 24.4 \\
\hline \multirow[t]{3}{*}{ Japan } & A & .. & .. & .. & .. & .. & 29.2 & 35.1 & 34.3 \\
\hline & B & .. &.. & .. & .. & .. & 3.9 & 6.1 & 4.3 \\
\hline & $\mathrm{C}$ & .. & .. & .. & .. & .. & 8.2 & 11.8 & 7.7 \\
\hline \multirow[t]{3}{*}{ Latvia } & A & .. &.. & .. & .. & .. & .. & 57.1 & 50.0 \\
\hline & B & .. & .. & .. & .. & .. & .. & 3.2 & 2.0 \\
\hline & $\mathrm{C}$ & .. &.. & .. & .. & .. & .. & 12.0 & 6.0 \\
\hline \multirow[t]{3}{*}{ Lithuania } & A & .. &.. & .. & .. & .. & 85.7 & 60.0 & 61.5 \\
\hline & B & .. &.. & .. & .. & .. & 13.2 & 5.3 & 4.6 \\
\hline & $\mathrm{C}$ &.. &.. &.. & .. &.. & 33.6 & 15.1 & 11.2 \\
\hline \multirow[t]{3}{*}{ Luxembourg } & A & .. &.. & .. & .. & .. & .. &.. &.. \\
\hline & B & .. & .. & .. & .. & .. & .. &.. &.. \\
\hline & $\mathrm{C}$ & .. &.. & .. & .. & .. &.. &.. &.. \\
\hline \multirow[t]{3}{*}{ Netherlands } & A & .. & 37.5 & 31.7 & .. & .. & 44.4 &.. & 23.8 \\
\hline & B & .. & 4.3 & 14.7 & .. & .. & 2.0 &.. & 0.9 \\
\hline & $\mathrm{C}$ & .. & 6.5 & 17.6 &.. &.. & 2.3 &.. & 1.0 \\
\hline
\end{tabular}




\begin{tabular}{|c|c|c|c|c|c|c|c|c|c|}
\hline \multirow[t]{2}{*}{ Country } & \multirow[t]{2}{*}{ Series } & \multicolumn{8}{|c|}{ Year } \\
\hline & & 1980 & 1985 & 1990 & 1995 & 2000 & 2005 & 2010 & 2015 \\
\hline \multirow[t]{3}{*}{ New Zealand } & A & .. & .. & 33.3 & 25.0 & 62.5 & 50.0 & 42.3 & 42.9 \\
\hline & B & .. & .. & 2.9 & 3.2 & 4.8 & 2.5 & 3.2 & 2.1 \\
\hline & $\mathrm{C}$ &.. & .. & 4.8 & 5.0 & 7.4 & 4.0 & 5.4 & 3.9 \\
\hline \multirow[t]{3}{*}{ Norway } & A & .. &.. & .. &.. &.. &.. &.. &.. \\
\hline & B & .. &.. & .. & .. &.. &.. &.. &.. \\
\hline & $\mathrm{C}$ & .. & .. & .. & .. &.. &.. &.. &.. \\
\hline \multirow[t]{3}{*}{ Poland } & A & .. &.. & .. & .. &.. & 15.4 & 50.0 & 40.0 \\
\hline & B & .. & .. & .. & .. & .. & 0.2 & 0.3 & 0.6 \\
\hline & $\mathrm{C}$ & .. & .. &.. & .. & .. & 0.2 & 0.5 & 1.1 \\
\hline \multirow[t]{3}{*}{ Portugal } & A & .. & .. & 50.0 & .. & 25.0 & 41.9 & 56.3 & 56.3 \\
\hline & B &.. & .. & 0.9 & .. & 1.0 & 1.3 & 1.3 & 4.6 \\
\hline & $\mathrm{C}$ & .. & .. & 2.8 & .. & 1.5 & 2.0 & 1.9 & 7.7 \\
\hline Slovak & A & .. & .. & .. & 41.8 & .. & 64.2 & 37.1 & 51.5 \\
\hline \multirow[t]{2}{*}{ Republic } & B & .. & .. & .. & 8.6 & 0.0 & 14.5 & 2.7 & 2.4 \\
\hline & $\mathrm{C}$ & .. &.. & .. & 37.0 & 0.0 & 24.1 & 5.5 & 5.3 \\
\hline \multirow[t]{3}{*}{ Slovenia } & A & .. & .. & .. & .. &.. & 100.0 & .. &.. \\
\hline & B &.. &.. &.. &.. &.. & 0.9 &.. &.. \\
\hline & $\mathrm{C}$ & .. & .. & .. & .. & .. & 1.5 & .. & .. \\
\hline \multirow[t]{3}{*}{ Spain } & A & .. &.. & 64.3 & 100.0 & 100.0 & 62.1 & 86.0 & 56.4 \\
\hline & B & .. &.. & 1.7 & 1.3 & 0.6 & 4.0 & 5.7 & 8.3 \\
\hline & $\mathrm{C}$ & .. & .. & 6.8 & 3.9 & 1.7 & 8.7 & 17.2 & 24.2 \\
\hline \multirow[t]{3}{*}{ Sweden } & A &.. &.. &.. & .. &.. &.. &.. &.. \\
\hline & B &.. &.. & .. & .. &.. &.. &.. &.. \\
\hline & $\mathrm{C}$ &.. &.. & .. & .. &.. &.. &.. &.. \\
\hline \multirow[t]{3}{*}{ Switzerland } & A &.. &.. &.. &.. &.. &.. &.. &.. \\
\hline & B &.. &.. & .. & .. &.. &.. &.. &.. \\
\hline & C &.. &.. &.. &.. &.. &.. &.. &.. \\
\hline \multirow[t]{3}{*}{ Turkey } & A &.. &.. &.. &.. & .. &.. & 27.3 & 22.2 \\
\hline & B & .. & .. & .. & .. &.. & .. & 0.4 & 0.4 \\
\hline & $\mathrm{C}$ & .. &.. & .. &.. & .. &.. & 0.8 & 1.1 \\
\hline United & A & .. & 38.6 & 33.8 & 36.5 & 39.6 & 37.6 & 52.5 & 51.4 \\
\hline \multirow[t]{2}{*}{ Kingdom } & B & .. & 1.9 & 2.6 & 3.9 & 2.4 & 1.6 & 3.7 & 3.2 \\
\hline & $\mathrm{C}$ & .. & 2.4 & 3.2 & 4.5 & 2.8 & 1.9 & 4.6 & 4.0 \\
\hline \multirow[t]{3}{*}{ United States } & A &.. &.. & .. & .. & 63.6 & 56.0 & 63.3 & 52.6 \\
\hline & B & .. &.. & .. & .. & 0.4 & 0.6 & 1.1 & 0.8 \\
\hline & $\mathrm{C}$ & .. &.. & .. & .. & 0.7 & 1.2 & 2.2 & 1.8 \\
\hline \multirow{3}{*}{$\begin{array}{l}\text { OECD } \\
\text { countries }\end{array}$} & A & .. & 37.9 & 43.0 & 51.3 & 39.9 & 33.5 & 40.0 & 38.9 \\
\hline & B & .. & 1.8 & 1.6 & 2.3 & 1.5 & 2.0 & 2.9 & 2.4 \\
\hline & $\mathrm{C}$ & .. & 3.2 & 2.9 & 4.0 & 2.8 & 3.8 & 5.4 & 4.5 \\
\hline
\end{tabular}




\begin{tabular}{|c|c|c|c|c|c|c|c|c|c|}
\hline \multirow[t]{2}{*}{ Country } & \multirow[t]{2}{*}{ Series } & \multicolumn{8}{|c|}{ Year } \\
\hline & & 1980 & 1985 & 1990 & 1995 & 2000 & 2005 & 2010 & 2015 \\
\hline European & A &.. & 44.6 & 44.0 & 40.5 & 36.5 & 41.7 & 51.0 & 44.0 \\
\hline \multirow[t]{2}{*}{ Union 22} & B & .. & 2.4 & 2.7 & 1.6 & 1.4 & 1.6 & 2.7 & 2.9 \\
\hline & $\mathrm{C}$ &.. & 4.6 & 5.5 & 2.9 & 2.2 & 2.4 & 3.9 & 4.2 \\
\hline European & A &.. & 44.6 & 44.0 & 40.5 & 36.5 & 43.5 & 50.6 & 44.9 \\
\hline \multirow[t]{2}{*}{ Union 28} & B & .. & 2.4 & 2.7 & 1.6 & 0.8 & 1.5 & 2.6 & 2.9 \\
\hline & $\mathrm{C}$ &.. & 4.6 & 5.5 & 2.9 & 1.6 & 2.5 & 4.0 & 4.3 \\
\hline \multirow[t]{3}{*}{ G7 countries } & A &.. & 35.4 & 42.0 & 53.3 & 42.0 & 32.2 & 39.7 & 37.6 \\
\hline & B & .. &.. &.. &.. & .. &.. & .. &.. \\
\hline & $\mathrm{C}$ &.. &.. &.. &.. &.. &.. &.. &.. \\
\hline \multirow[t]{3}{*}{ Bulgaria } & A & .. & .. & .. &.. & .. & 61.5 & 51.9 & 77.8 \\
\hline & B & .. & .. & .. &.. & .. & 9.4 & 11.6 & 6.2 \\
\hline & $\mathrm{C}$ &.. & .. &.. & .. & .. & 24.2 & 53.7 & 28.8 \\
\hline \multirow[t]{3}{*}{ Croatia } & A &.. & .. &.. & .. & .. &.. & 100.0 &.. \\
\hline & B &.. &.. &.. &.. &.. &.. & 0.5 &.. \\
\hline & $\mathrm{C}$ & .. & .. & .. &.. & .. & .. & 0.6 & .. \\
\hline \multirow[t]{3}{*}{ Cyprus } & A & .. &.. &.. &.. & .. & 66.7 & 25.0 & 33.3 \\
\hline & B &.. & .. &.. &.. & .. & 8.8 & 2.9 & 4.5 \\
\hline & $\mathrm{C}$ &.. & .. &.. & .. & .. & 13.6 & 5.2 & 7.5 \\
\hline FYR of & A &.. &.. &.. &.. &.. &.. &.. & 100.0 \\
\hline \multirow[t]{2}{*}{ Macedonia } & B &.. &.. &.. &.. &.. &.. &.. & 2.9 \\
\hline & $\mathrm{C}$ & .. & .. &.. &.. & .. & .. &.. & 9.3 \\
\hline \multirow[t]{3}{*}{ Malta } & A &.. &.. & .. &.. & .. &.. &.. &.. \\
\hline & B & .. &.. &.. &.. & .. & .. & .. & .. \\
\hline & $\mathrm{C}$ &.. & .. &.. & .. & .. & .. & .. & .. \\
\hline \multirow[t]{3}{*}{ Romania } & A &.. &.. & .. &.. & .. & 36.4 & 44.9 & 51.0 \\
\hline & B &.. & .. &.. &.. & .. & 0.3 & 1.0 & 1.6 \\
\hline & $\mathrm{C}$ &.. &.. & .. &.. &.. & 1.0 & 2.7 & 3.7 \\
\hline Russian & A &.. & .. &.. & 23.8 & 43.9 & 70.4 & 59.9 & 78.0 \\
\hline \multirow[t]{2}{*}{ Federation } & B &.. &.. &.. & 0.7 & 0.2 & 0.6 & 1.1 & 1.6 \\
\hline & $\mathrm{C}$ &.. & .. &.. & 5.3 & 0.6 & 1.7 & 3.4 & 5.2 \\
\hline \multirow[t]{3}{*}{ Colombia } & A &.. &.. & .. &.. &.. & 40.5 & 34.8 & 40.4 \\
\hline & B &.. & .. &.. & .. &.. & 7.7 & 7.0 & 6.7 \\
\hline & $\mathrm{C}$ &.. &.. &.. &.. &.. & 18.0 & 13.5 & 12.9 \\
\hline
\end{tabular}

Source: OECD.Stat.

Table 1.10 presents the equivalent data for women aged 65 or over. Here, it is evident that this group of women's situation is somewhat different. As opposed to their younger counterparts, such women represent a minority of involuntary part-time workers and, in some countries, substantially so, but here wide variations between nations are also evident. 
Similar to their younger counterparts, this group of women represents a growing, yet modest, proportion of all those in employment. Overall, they represent a modest share of part-time workers, with exceptions being Chile, Italy, Spain and Bulgaria. Thus, it is evident that such work does not generally represent as much of a problem for this age group as it does for some of their younger counterparts.

Table 1.11 Numbers of women in different industry sectors by age group for Australia

\begin{tabular}{|c|c|c|c|c|}
\hline \multirow[t]{2}{*}{ Industry sector } & \multicolumn{4}{|c|}{ Age group } \\
\hline & $55-59$ & $60-64$ & $65-69$ & $70-74$ \\
\hline Agriculture, Forestry and Fishing & 9090 & 7784 & 6425 & 4139 \\
\hline Mining & 1665 & 789 & 242 & 72 \\
\hline Manufacturing & 18882 & 11291 & 4164 & 1237 \\
\hline Electricity, Gas, Water and Waste Services & 2134 & 1070 & 300 & 78 \\
\hline Construction & 10325 & 6390 & 2731 & 890 \\
\hline Wholesale Trade & 9037 & 5437 & 2259 & 662 \\
\hline Retail Trade & 44108 & 27548 & 11439 & 3356 \\
\hline Accommodation and Food Services & 19663 & 12210 & 4870 & 1526 \\
\hline Transport, Postal and Warehousing & 12090 & 7450 & 3013 & 934 \\
\hline Information Media and Telecommunications & 4190 & 2723 & 1119 & 355 \\
\hline Financial and Insurance Services & 13401 & 7564 & 2595 & 735 \\
\hline Rental, Hiring and Real Estate Services & 7547 & 5224 & 2869 & 1131 \\
\hline Professional, Scientific and Technical Services & 23891 & 15872 & 7961 & 2791 \\
\hline Administrative and Support Services & 18523 & 12508 & 5398 & 1770 \\
\hline Public Administration and Safety & 33554 & 18907 & 6378 & 1516 \\
\hline Education and Training & 74989 & 47577 & 17165 & 4553 \\
\hline Health Care and Social Assistance & 122111 & 79298 & 29780 & 7434 \\
\hline Arts and Recreation Services & 5403 & 3599 & 1931 & 769 \\
\hline Other Services & 13850 & 9270 & 4183 & 1477 \\
\hline
\end{tabular}

Source: Census of Population and Housing, 2016, TableBuilder.

At the same time as it is argued older women's relationship with the labour market is transforming, it is important to note that some industry sectors' workforces are older than others and some sectors remain highly gendered. Considering issues of where older women work, international comparisons are not available or the data are rather dated. Thus, the following brief discussion is focused on the case of Australia.

Table 1.11 shows the industry sectors in which older women work, drawing on Australian Census data for 2016. This indicates the magnitude of older women's contribution to the Australian economy, with approximately 900000 women aged 55-74 working across a range of industries. It is apparent from this table that sectors where women over the age of 50 predominate are, from highest, Health Care and Social Assistance; Education and Training; Retail Trade; and Public Administration and Safety. Participation in these industry sectors alone vastly outweighs participation in other sectors. This pattern of participation applies across older age groups. 
Notably, around 250000 women aged between 55 and 74 work in Health Care and Social Assistance, while around 150000 work in Education and Training. Thus, there is potential value in considering industry sector when researching and devising public policy for older women.

Table 1.12 Numbers of women in different occupational groups by age group for Australia

\begin{tabular}{lllll}
\hline \multicolumn{1}{c}{ Occupational group } & \multicolumn{3}{c}{ Age group } \\
\cline { 2 - 5 } & $55-59$ & $60-64$ & $65-69$ & $70-74$ \\
\hline Managers & 49012 & 29933 & 15196 & 6492 \\
Professionals & 112318 & 71812 & 27696 & 7793 \\
Technicians and Trades Workers & 17103 & 10326 & 4000 & 1263 \\
Community and Personal Service Workers & 67492 & 40748 & 14418 & 3752 \\
Clerical and Administrative Workers & 117387 & 77131 & 33297 & 10169 \\
Sales Workers & 40399 & 26219 & 11199 & 3288 \\
Machinery Operators and Drivers & 8096 & 4839 & 1617 & 498 \\
Labourers & 41646 & 27852 & 10632 & 3591 \\
Inadequately described & 3388 & 2080 & 1010 & 424 \\
Not stated & 2220 & 1761 & 1284 & 1025 \\
\hline
\end{tabular}

Source: Census of Population and Housing, 2016, TableBuilder.

Table 1.12 shows the occupational groups in which Australian older women work. It is noteworthy that over 100000 managers in Australia are women aged 55-74. It is apparent that Professionals along with Clerical and Administrative Workers predominate, this pattern being reflected across age groups. Also noticeable are the numbers of older women described as Labourers. Of these approximately 14500 are aged 65 and over, of which, notably, over 3500 are aged over 70. Thus, older women participate in the Australian labour force at all levels, making the notion of a one-size-fits-all policy approach problematical.

\section{DISCUSSION}

This chapter has reviewed trends in older women's labour force participation internationally to show that patterns are uneven and there are variations across countries. This makes it difficult to describe a single story of older women's relationship with paid work. A substantial proportion of older women engage in full-time paid work. However, as we show, others also continue to work, including in fixed-term and casual roles and at reduced hours when compared to career employment. As we reveal in later chapters, some women do not experience any dramatic transformation as they transition from working to retirement, and they continue to juggle a range of paid and unpaid activities and responsibilities before and during retirement. 